\title{
Excited-state quantum phase transitions in a two-fluid Lipkin model
}

\author{
J.E. García-Ramos ${ }^{1}$, P. Pérez-Fernández², J.M Arias $^{3}$ \\ ${ }^{1}$ Departamento de Ciencias Integradas, \\ Universidad de Huelva, 21071 Huelva, \\ Spain and Instituto Carlos I de Física Teórica y Computacional, \\ Universidad de Granada, Fuentenueva s/n, 18071 Granada, Spain \\ ${ }^{2}$ Departamento de Fúsica Aplicada III, \\ Escuela Técnica Superior de Ingeniería, \\ Universidad de Sevilla, Sevilla, Spain \\ ${ }^{3}$ Departamento de Física Atómica, Molecular y Nuclear, \\ Facultad de Física, Universidad de Sevilla, \\ Apartado 1065, 41080 Sevilla, Spain
}




\begin{abstract}
Background: Composed systems have became of great interest in the framework of the ground state quantum phase transitions (QPTs) and many of their properties have been studied in detail. However, in these systems the study of the so called excited-state quantum phase transitions (ESQPTs) have not received so much attention.

Purpose: A quantum analysis of the ESQPTs in the two-fluid Lipkin model is presented in this work. The study is performed through the Hamiltonian diagonalization for selected values of the control parameters in order to cover the most interesting regions of the system phase diagram.
\end{abstract}

Method: A Hamiltonian that resembles the consistent-Q Hamiltonian of the interacting boson model (IBM) is diagonalized for selected values of the parameters and properties such as the density of states, the Peres lattices, the nearest-neighbor spacing distribution, and the participation ratio are analyzed.

Results: An overview of the spectrum of the two-fluid Lipkin model for selected positions in the phase diagram has been obtained. The location of the excited-state quantum phase transition can be easily singled out with the Peres lattice, with the nearest-neighbor spacing distribution, with Poincaré sections or with the participation ratio.

Conclusions: This study completes the analysis of QPTs for the two-fluid Lipkin model, extending the previous study to excited states. The ESQPT signatures in composed systems behave in the same way as in single ones, although the evidences of their presence can be sometimes blurred. The Peres lattice turns out to be a convenient tool to look into the position of the ESQPT and to define the concept of phase in the excited states realm.

PACS numbers: 21.60.Fw, 02.30.Oz, 05.70.Fh, 64.60.F-

Keywords: Lipkin model, two-fluid system, excited-state quantum phase transitions 


\section{INTRODUCTION}

During almost twenty years, quantum phase transitions (QPTs) have been a hot topic in different areas of quantum many-body physics. On one hand, QPTs in Nuclear Physics have been deeply studied [1 3], from both theoretical and experimental points of view. On the other hand, other fields such as Molecular Physics [4, 5], Quantum Optics [6, 7] or Solid State Physics [8] put forward the QPTs studies.

The well known thermodynamic phase transitions develop in systems with an infinite number of particles, i.e., in the thermodynamic limit, in this sense they are called classical phase transitions. QPTs are phenomena similar to classical phase transitions but differ in that QPTs take place at zero temperature. In a broad sense, QPTs appear in Hamiltonians that can be split into two parts, each of them presenting a different symmetry. In this situation a simple transitional Hamiltonian can be written as a function of one control parameter that governs the change in the system from one symmetry to the other,

$$
H(\xi)=\xi \cdot H\left(\text { symmetry }_{1}\right)+(1-\xi) \cdot H\left(\text { symmetry }_{2}\right) .
$$

The phase of the system is characterized by a parameter, usually called order parameter, that is zero in one phase and different from zero in the other. A QPT is characterized by a sudden change in the value of the order parameter for a small variation around a particular value, $\xi_{c}$, of the control parameter, $\xi$. The value $\xi_{c}$ where the QPT develops is known as the critical point and marks when the system undergoes a structural change from symmetry ${ }_{1}$ to symmetry $_{2}$.

An appealing step forward in the QPT concept is its extension to composed systems, i.e., systems with different species of components. The simplest case is a quantum system with two of such species or fluids. One interesting case is the composed boson-fermion system [9 11], although here we will focus in a two fluid model in which the two species are bosons and are represented by creation and annihilation boson operators that fulfill the usual boson commutation relations. In this framework, it is worth to mention the very first studies in Nuclear Physics of two-fluid systems [12 14], conducted for the proton-neutron interacting boson model. In similar schemes, the bending dynamics of tetratomic molecules has also been studied with a two-fluid bosonic model where each fluid is associated with a bender [15, 16]. Other two-fluid systems, that still today act as landmarks, are the Dicke [17] and the Jaynes-Cumming [18] models for which the two fluids correspond to photons 
and atoms. Finally, another simple composed model is the two-fluid Lipkin model [19], where the fluids may correspond to two species of atoms or to two vibrational modes. Dicke and Jaynes-Cumming models are algebraically connected with the two-fluid Lipkin model. Indeed, the dynamical algebra of the double Lipkin model is $u(2) \otimes u(2)$ and one can go to Dicke and Jaynes-Cumming models through a contraction from the $u(2)$ Lie algebra to the Heisenberg-Weyl one $h w(1)$ [20].

The aim of this work is to extend the study of the two-fluid Lipkin model, whose QPTs and phase diagram were studied in detail in [21, to the excited states realm, in other words, to study the excited-state quantum phase transitions (ESQPTs) of the model. The term ESQPT was first coined in [22] and studied in detail in [23]. In [23] it is stated that "The infinite level density, moreover, propagates to higher excitation energy ... hence the concept of a continuation of the QPT to excited states". Consequently, an ESQPT can be understood as the propagation of the QPT to excited states. Moreover, an ESQPT is deeply connected to the existence of a barrier in the potential energy surface and, in particular, with the height of the barrier. The presence of an ESQPT is marked by the presence of a singularity in the density of states, but it is also known to affect the structure of the states. In fact, these change, from being non-symmetric or deformed to symmetric or spherical (or viceversa), when crossing the ESQPT. Another relevant fact of the ESQPT is that it seems to be related to a change from a regular to a chaotic regime, although this point is still an open question.

In this manuscript, the study of the phase diagram for the two-fluid Lipkin model is completed by extending the work of Ref. [21] to the excited states. The paper is organized as follows: in Section II the algebraic structure and the model Hamiltonian are outlined. In Section IIB the main ingredients of its phase diagram are revised. In Section III the ESQPT concept is introduced and, in particular, the main tools used to study the onset of an ESQPT are commented. In Section IV the main outcome of this work is presented and we study in detail the onset of ESQPTs in some particular cases. Finally, Section V stands for the summary and conclusions. 


\section{THE LIPKIN MODEL AND ITS TWO-FLUID EXTENSION}

The Lipkin model was proposed in the 1960's by Lipkin, Meshkov, and Glick [19] as a simple exactly solvable model to check the validity and limitations of different approximation methods used in Nuclear Physics. Since then, the model has been applied to other fields and many examples of its use can be found in the literature.

\section{A. Algebraic structure}

Using a boson representation, the Lipkin model is built in terms of scalar bosons that can occupy two non-degenerated energy levels labeled by $s$ and $t$. In the case of a single fluid, the building blocks are the creation $s^{\dagger}, t^{\dagger}$, and annihilation $s, t$, boson operators. The four possible bilinear products of one creation and one annihilation boson operator generate the $u(2)$ algebra. The next step to obtain the two-fluid Lipkin model is to combine two coupled Lipkin structures. In this model, there are two boson families identified by a subindex, $s_{1}^{\dagger}, t_{1}^{\dagger}$ and $s_{2}^{\dagger}, t_{2}^{\dagger}$, and the corresponding dynamical algebra will be $u_{1}(2) \otimes u_{2}(2)$, whose generators are: $s_{i}^{\dagger} s_{i}, s_{i}^{\dagger} t_{i}, t_{i}^{\dagger} s_{i}$, and $t_{i}^{\dagger} t_{i}$, for $i=1,2$.

A detailed description of the $u_{1}(2) \otimes u_{2}(2)$ algebraic structure can be found in [24]. Here, we simply summarize some features that will be of interest along this work. Starting from the dynamical algebra $u_{1}(2) \otimes u_{2}(2)$, the possible subalgebras chains are four. However, for us only two of them, in which there is an early coupling of the dynamical algebras into the direct-sum subalgebra $u_{12}(2)$ (or $s u_{12}(2)$ ), are relevant,

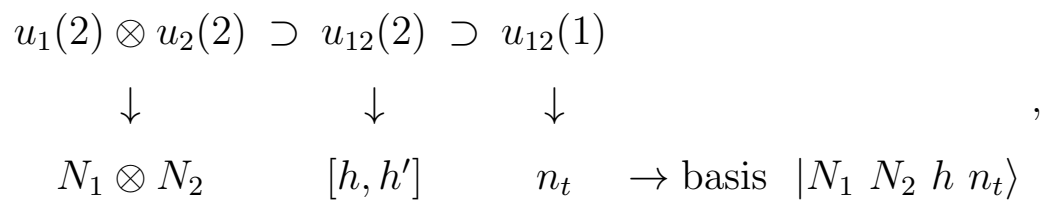

where the labels of the irreps verify the following branching rules: $h+h^{\prime}=N_{1}+N_{2}, h \geq h^{\prime}$, $1 / 2\left(N_{1}+N_{2}+h^{\prime}-h\right) \leq n_{t} \leq 1 / 2\left(N_{1}+N_{2}+h^{\prime}-h\right)$, and

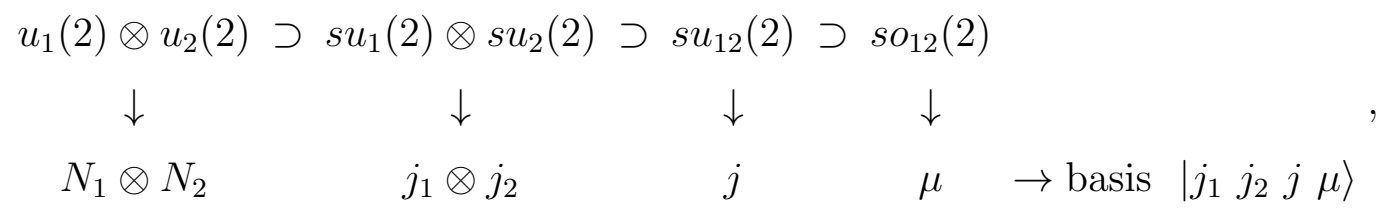

where $j_{i}=N_{i} / 2, j=1 / 2\left(N_{1}+N_{2}\right), 1 / 2\left(N_{1}+N_{2}\right)-1, \ldots, 1 / 2\left|N_{1}-N_{2}\right|,-j \leq \mu \leq j$, and $j=1 / 2\left(h-h^{\prime}\right) .|\ldots\rangle$ stands for the basis state in the corresponding dynamical symmetry. 
The Hamiltonian used in this work is inspired in the consistent-Q-formalism of the interacting boson model [25]. The Hamiltonian can be written as,

$$
H=x\left(n_{t_{1}}+n_{t_{2}}\right)-\frac{1-x}{N_{1}+N_{2}} Q^{\left(y_{1}, y_{2}\right)} \cdot Q^{\left(y_{1}, y_{2}\right)}
$$

where

$$
\begin{aligned}
n_{t_{i}} & =t_{i}^{\dagger} t_{i}, \\
Q^{\left(y_{1}, y_{2}\right)} & =\left(Q_{1}^{y_{1}}+Q_{2}^{y_{2}}\right), \\
Q_{i}^{y_{i}} & =s_{i}^{\dagger} t_{i}+t_{i}^{\dagger} s_{i}+y_{i}\left(t_{i}^{\dagger} t_{i}\right) .
\end{aligned}
$$

The Hamiltonian (4) has three control parameters $\left(x, y_{1}\right.$, and $\left.y_{2}\right)$. The model has two order parameters $\beta_{1}$ and $\beta_{2}$ associated to each fluid: $\beta_{i}=0$ values indicate the symmetric or spherical phase for fluid $i$, while values different from zero characterize non-symmetric or deformed phases. Due to the behaviour of the bosons under parity, the Hamiltonian (4) is, in general, non-parity conserving, except for $y_{1}=y_{2}=0$.

The Hamiltonian (4) is a mixture of dynamical symmetries of the problem, particularly $u_{12}(1)$ for $x=1$, and $s o_{12}(2)$ for $x=0$ and $y_{1}=y_{2}=0$. This form is specially suitable to study QPTs, because one can associate a symmetric (spherical) phase to the first term of the Hamiltonian and a non-symmetric (deformed) shape to the second term. Moreover, depending on the values of $y_{1}$ and $y_{2}$, different kinds of deformation are generated. For us, it is specially important the case $y_{1}=y_{2}$, for which the dynamical algebra of the Hamiltonian will be $u_{12}(2)\left(s u_{12}(2)\right)$ and the states will belong to a single $\left[h, h^{\prime}\right]$ Young tableau (with a well defined value of $j$ ). In this case, one can separate the spectrum in families with given $j$-values. Note that although $j$ has the properties of an angular momentum, it is not an orbital angular momentum in the sense of $L$ in the interacting boson model or in the vibron model. It is, indeed, similar to the concept of F-spin in the proton-neutron interacting boson model [26].

\section{B. The phase diagram}

The combination of numerical calculations with analytical results, as shown in [21], provides the phase diagram depicted in Fig. 1. In that reference an essential order parameter $\beta=\left(\beta_{1}+\beta_{2}\right) / 2$ is defined and it is the parameter that characterizes the different phases: 


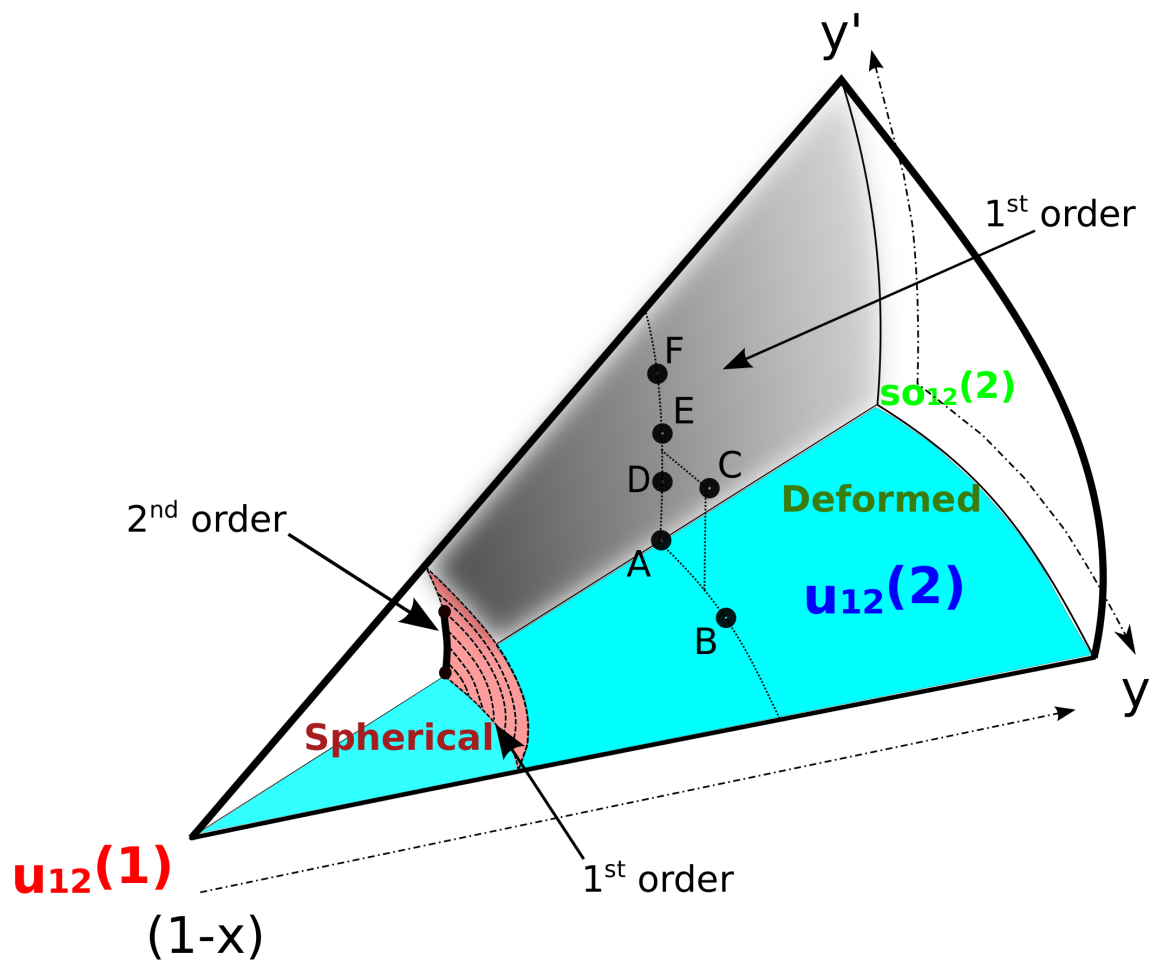

FIG. 1: Phase diagram of the consistent-Q like two-fluid Lipkin model. In the diagram the different phases are represented: spherical and deformed, the first order QPT surfaces and the second order QPT line. Moreover the relevant control parameters $\left(x, y=\left(y_{1}+y_{2}\right) / 2\right.$, and $\left.y^{\prime}=\left(y_{1}-y_{2}\right) / 2\right)$ and dynamical symmetries also are shown. The marked points correspond to the cases studied in Section IV (see text).

spherical means $\beta=0$, while two different deformations can appear, $\beta>0$ and $\beta<0$. Note that the order parameter $\beta$ is equivalent to $\beta_{a}$ as it appears in Ref.[21].

The Hamiltonian (4) can be more conveniently rewritten in terms of control parameters $y=\left(y_{1}+y_{2}\right) / 2$, and $y^{\prime}=\left(y_{1}-y_{2}\right) / 2$. In Fig. 1 the phase diagram of the model in the space of coordinates $x, y$, and $y^{\prime}$ is presented. There, a first order phase transition surface (in red) separates the symmetric (spherical, $\beta=0$ ) and non-symmetric (deformed) phases (the one shown here corresponds to $\beta>0$ ). This first order phase transition region appears in many other models (quantum cusp, interacting boson model, Dicke and Jaynes-Cumming, among others) and essentially stems from the competition between single particle terms that lead to spherical shapes and two-body interactions that lead to deformed configurations. Therefore, this phase transition is related with the evolution and competition of the spherical and the deformed minima (there is a region of coexistence of minima and the critical point is defined 
by the degeneration of both minima). This surface contains a line (black thick line in the figure) that runs from $y=y^{\prime}=0$ to $y=0, y^{\prime}=1$ and corresponds to a second order phase transition. This line is, in fact, a triple point where three degenerated minima coexist (one spherical, and two deformed with different deformation). The point $y=0, y^{\prime}=1$ shows a unique behaviour because presents a divergence [21] in the second derivative of the energy with respect to the control parameter. At this point, the spinodal and the antispinodal lines merge with the first order phase transition surface giving rise to a tricritical point.

The phase diagram shown in Fig. 1 can be completed by extending it to negative values of $y$ and $y^{\prime}$. In particular the negative $y$ values imply deformation $\beta<0$. The vertical surface (in grey), separates deformed phases with different signs in the value of the order parameter $\beta$. This is a first order phase transition surface. This phase transition is connected with the existence of two deformed minima, $\beta<0$ and $\beta>0$, that eventually can become degenerated (the grey surface in the figure). Note that in this situation, the potential is symmetric under an appropriate interchange of the two shape variables $\left(\beta_{1}\right.$ and $\beta_{2}$, order parameters). At each side of the surface two deformed minima coexist but in the side shown in Fig. 1 the absolute minimum corresponds to $\beta>0$ while at the other side the absolute minimum appears for $\beta<0$. This situation is different to what happens at the phase transition surface separating spherical $(\beta=0)$ and deformed $(\beta>0)$ phases. In this case, the system transits from a single spherical minimum, $\beta=0$, to a deformed minimum $(\beta>0)$ through a coexistence region. In the interacting boson model the first situation corresponds to the QPT between the $S U(3)$ and the $\overline{S U(3)}$ limits [27], while the second corresponds to the QPT appearing when passing from the $U(5)$ limit to the $S U(3)-O(6)$ line [26].

\section{EXCITED-STATE QUANTUM PHASE TRANSITIONS}

In many-body quantum systems the presence of a ground state QPT can give rise to an ESQPT when using the excitation energy as a control parameter [23]. Once in the deformed phase, keeping the control parameters fixed, one can go up in energy and look into a magnitude that marks the presence of a quantum phase transition in the excited states. This magnitude can be the density of states which is expected to have some kind of singularity when reaching the energy at which an ESQPT develops. In particular, this

is the case for the one-fluid Lipkin model, where the QPT for the ground state seems to 
"propagate" to the excited states. An ESQPT associated to second order QPT is defined as a singularity in the density of states or in one of its derivatives. The kind of singularity depends on the number of degrees of freedom of the system in the semiclassical limit [28, 29]. In particular, the Lipkin model, which has a single degree of freedom, presents a $\lambda$ singularity in the density of states at the excitation energy corresponding to the ESQPT. The Dicke model, with two degrees of freedom, presents at the ESQPT critical energy a discontinuity in the first derivative of the density of states [28]. The sudden increase in the density of states is related with the presence of a maximum in the potential energy surface of the system. Starting from the bottom of the potential the states bunch up when reaching the maximum of the potential giving rise to an increase of the density of states. On the other hand, when the QPT is of first order, besides the previous behaviour, linked to the existence of a maximum, the presence of a new family of states related with the existence of an extra local minimum induces a finite increase in the density of states and, therefore, a discontinuity in the density of states. The energy at which the second family of states appears in the spectrum corresponds to the energy of the second minimum.

To explain more in detail the connection between the presence of a maximum in the potential energy surface and the onset of an ESQPT, we present in Fig. 2 two calculations for large number of bosons, $N=1000$, evaluating the density of states for the single Lipkin Hamiltonian. In panel (a) we perform a calculation for a Hamiltonian in the deformed phase. The Hamiltonian represents a situation in which the ground state is deformed but comes from the evolution of a spherical ground state through a second order phase transition, this means that there is a maximum at zero deformation (see inset of Fig. 2a). Note that there are, in fact, two degenerated minima that give rise to degenerated doublets below the ESQPT. The existence of the maximum induces many states bunching together in the spectrum, leading, therefore, to a sudden increase (a singularity in the thermodynamic limit) in the density of states at the energy of the maximum (this is seen at zero energy in panel (a)). In order to show the difference with the case of a first order phase transition, in panel (b) a calculation of the density of levels is presented for a Hamiltonian owning a deformed ground state that now comes from the evolution of a spherical ground state through a first order phase transition. This means that at the QPT there is coexistence of spherical and deformed minima. This fact has as a consequence that far from the QPT, in the deformed phase, the system presents a local deformed minimum too, being both deformed minima 
separated by the spherical maximum (this can be seen in the inset of Fig. 2b). Starting from the bottom of the potential and going up in energy, when the energy of the local minimum is reached, a new family of states appears and it produces a finite increase in the density of states (at energy around -200 in panel (b)). Going upper in energy one reaches the maximum of the potential and a new bunching up of the energy levels is observed in the spectrum, producing a peak in the density of states (at zero energy in panel (b)), this will give a lambda singularity in the thermodynamic limit).
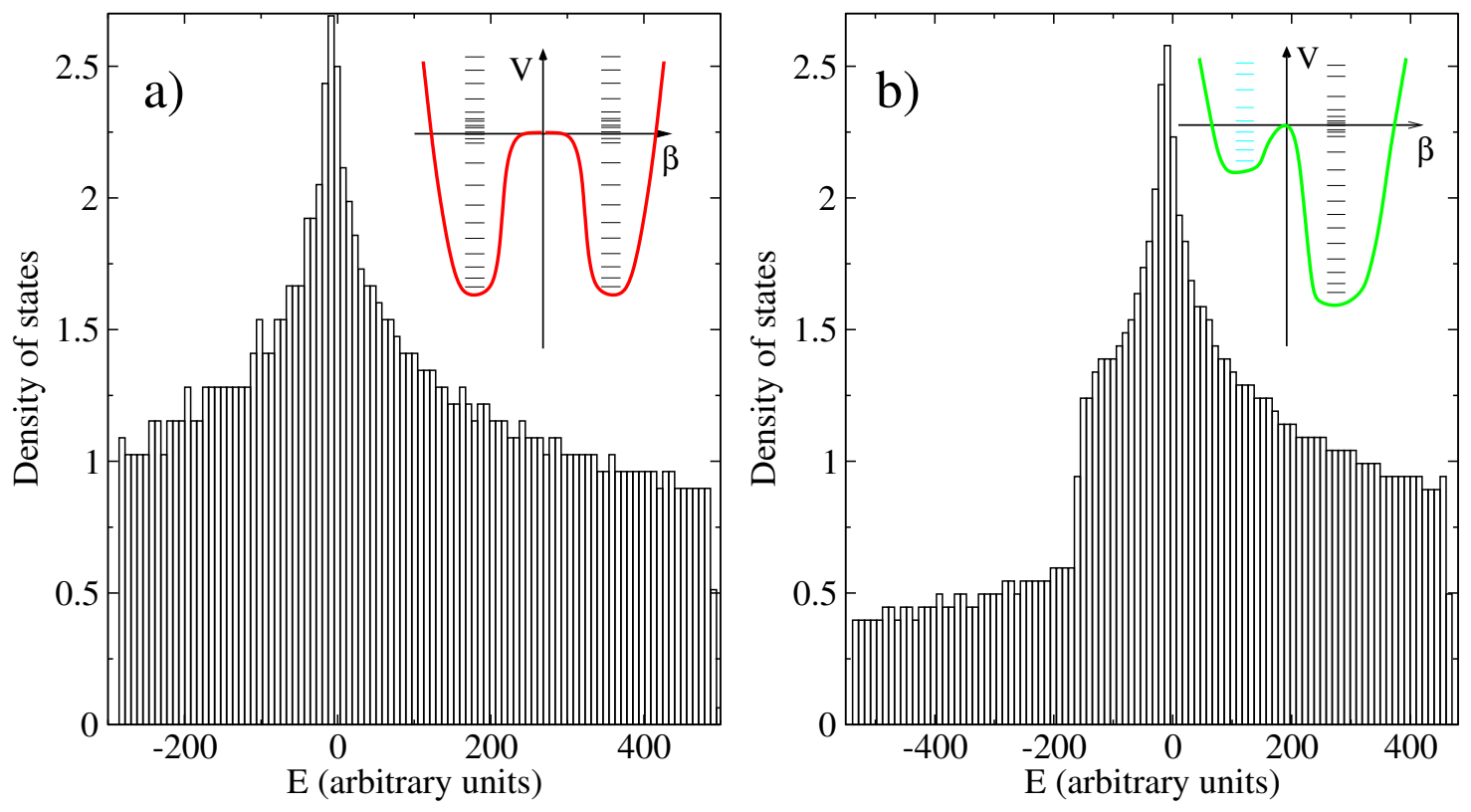

FIG. 2: Density of states for a one-fluid Lipkin Hamiltonian $H=x n_{t}-\frac{1-x}{N} Q^{(y)} \cdot Q^{(y)}\left(n_{t}\right.$ and $Q^{(y)}$ defined in (5) and (7) with $x=1 / 2, y=0$, and $N=1000$ (panel (a)) and with $x=1 / 2$, $y=1 / 2$, and $N=1000$ (panel (b)); the insets show schematically the corresponding potential energy surface in both cases.

Let us now come to our case of interest, the two-fluid Lipkin model given by Eq. (4). The Hamiltonian is such that for any set of parameter values that lead to a deformed ground state, the energy potential has always a maximum at $\beta=0$. The reason is the absence of linear terms in the potential (see Eq. (27) of [21]). This also happens in the interacting boson model. Therefore, for such a situation, it appears in the spectrum an ESQPT that is related with the existence of a maximum in the potential energy surface at $\beta=0$. As a consequence, for our Hamiltonian, the critical energy of the ESQPT will be always zero because it corresponds to the value of the potential energy surface at $\beta=0$. 
Although the concept of phase is strictly defined for the ground state, one can extend it to the excited states [23], in the sense that the excited states resemble the ground state with $\beta=0$ (symmetric states) or with $\beta \neq 0$ (non-symmetric states). As a matter of fact, in Fig. 11 of Ref. [23] one can clearly see how the states can be divided into two families, each one with reminiscences of one of the different Hamiltonian symmetries (see Hamiltonian (1)). The states below the ESQPT energy can be assigned to the non-symmetric (deformed) phase, while the ones above the ESQPT energy are in the symmetric (spherical) phase, or viceversa.

\section{A. Some tools to study ESQPTs}

As we have already explained, an ESQPT has been mainly identified by some kind of singularity in the density of states. However, this is neither the only signal of the presence of an ESQPT, nor the most efficient, specially for the study of finite size systems. In this section, a set of quantities that could serve as markers for ESQPT's are briefly discussed.

\section{Density of states}

This is the most obvious tool to reveal the presence of an ESQPT, however with this observable the presence of an ESQPT is not always so clear as in Fig. 2, For example, in the case of systems with two degrees of freedom, the discontinuity appears in the derivative of the density of states and, therefore, it is hard to be detected in a numerical calculation. The size of the system is also an issue because it could hide the presence of an ESQPT. For instance, a low number of states will hinder the detection of an ESQPT in any numerical calculation of density of states. Finally, the existence of an unnoticed symmetry in the Hamiltonian will change completely the observed behaviour in Fig. 2 and the density of states will correspond to the superposition of multiple belts with smoothed singularities at an energy that is shifted, making much harder to notice any singularity. 
2. Chaotic versus regular behaviour: Nearest-neighbor spacing distribution, Poincaré sections, and Peres lattices

The interplay between ESQPTs and the onset of chaos has been studied in [30 32]. It has been shown that, in particular cases, but not in general, the behaviour of the nearestneighbor spacing distribution (NNSD) suddenly changes from regular to chaotic distribution when crossing the ESQPT energy. The NNSD, thus, could be used as a signature of the presence of an ESQPT at a given energy. However, it will become specially effective if the character, either regular or chaotic, changes for all the states at roughly the same energy, and there are no regular states coexisting with the chaotic ones. The reason is that the NNSD provides a survey that is not local but only valid within a certain range of energy.

For the Hamiltonian under study, the NNSD is not an ideal tool because, as it will be shown, regular and chaotic states are not well separated in the spectrum. However, as we will see, one can still get a clear signal when crossing the energy of the ESQPT. To use the NNSD as a hint for the presence of chaos we should remember that in integrable systems the states follow a Poisson distribution $P_{P}(s)=\exp (-s)$, while in fully chaotic ones, they follow a Wigner distribution $P_{W}(s)=\frac{\pi}{2} s \cdot \exp \left(-\frac{\pi}{4} s^{2}\right)$, where $s_{i}=\left(E_{i+1}-E_{i}\right) /\langle s\rangle$ is a normalized distance between levels. We will measure the degree of chaos defining the quantity

$$
\eta=\frac{\sigma_{s}-\sigma_{W}}{\sigma_{P}-\sigma_{W}}
$$

where $\sigma_{s}=\left\langle s^{2}\right\rangle-\langle s\rangle^{2}$ is the variance of the analyzed spectrum, $\sigma_{W}=4 / \pi-1$ is the variance of the Wigner distribution, and $\sigma_{P}=1$ is the variance of the Poisson distribution. Therefore, the system will be fully regular for $\eta=1$, and fully chaotic for $\eta=0$. It is worth noting that in order to calculate $\eta$ all the considered states should have the same symmetry, e.g., in the case of states with given parity, all the states should have the same parity. To mark the onset of chaos one can plot the value of $\eta$, calculated for a certain number of states, as a function of the energy.

Another option to study the onset of chaos is to build the classical counterpart of Hamiltonian (4) and to study its semiclassical dynamics [29]. To this end we define a coherent state as the one of Ref. [21], but taking the deformation parameter, $\beta_{k}$, as complex and redefining it, such that its absolute value is constrained to the interval $[0,1], \tilde{\beta}_{k} \tilde{\beta}_{k}^{*}=\beta_{k}^{2} /\left(1+\beta_{k}^{2}\right)$,

$$
\left|\tilde{\beta}_{1}, \tilde{\beta}_{2}\right\rangle=\frac{1}{\sqrt{N_{1} ! N_{2} !}}\left(\sqrt{1-\tilde{\beta}_{1} \tilde{\beta}_{1}^{*}} s_{1}^{\dagger}+\tilde{\beta}_{1} t_{1}^{\dagger}\right)^{N_{1}}\left(\sqrt{1-\tilde{\beta}_{2} \tilde{\beta}_{2}^{*}} s_{2}^{\dagger}+\tilde{\beta}_{2} t_{2}^{\dagger}\right)^{N_{2}}|0\rangle .
$$


The classical limit of the system is then given by the expectation value of the Hamiltonian:

$$
H_{c l}\left(\tilde{\beta}_{1}, \tilde{\beta}_{1}{ }^{*}, \tilde{\beta}_{2}, \tilde{\beta}_{2}{ }^{*}\right)=\left\langle\tilde{\beta}_{1}, \tilde{\beta}_{2}|H| \tilde{\beta}_{1}, \tilde{\beta}_{2}\right\rangle .
$$

From $\tilde{\beta}_{1}$ and $\tilde{\beta}_{2}$ one can define the canonical variables $\left(q_{1}, p_{1}\right)$ and $\left(q_{2}, p_{2}\right)$, such that they verify:

$$
\tilde{\beta}_{k}=\frac{1}{\sqrt{2}}\left(q_{k}+\imath p_{k}\right),
$$

with $k=1,2$. Therefore, the classical Hamiltonian per particle, $h_{c l}\left(q_{1}, p_{1}, q_{2}, p_{2}\right)=$ $H_{c l}\left(q_{1}, p_{1}, q_{2}, p_{2}\right) / N$, for a system with $N_{1}=N_{2}$ can be written as,

$$
\begin{aligned}
h_{c l}\left(q_{1}, p_{1}, q_{2}, p_{2}\right) & =\frac{x}{4}\left(p_{1}^{2}+p_{2}^{2}+q_{1}^{2}+q_{2}^{2}\right)+\frac{x-1}{16}\left(2 q _ { 1 } ^ { 2 } \left(p_{1}^{2}\left(y_{1}^{2}-2\right)\right.\right. \\
& \left.+2 q_{2} y_{1} \sqrt{-p_{2}^{2}-q_{2}^{2}+2}+p_{2}^{2} y_{1} y_{2}+q_{2}^{2} y_{1} y_{2}+4\right) \\
& +4 q_{1} \sqrt{-p_{1}^{2}-q_{1}^{2}+2}\left(p_{1}^{2} y_{1}+2 q_{2} \sqrt{-p_{2}^{2}-q_{2}^{2}+2}+p_{2}^{2} y_{2}+q_{2}^{2} y_{2}\right) \\
& +2 q_{2}^{2}\left(p_{1}^{2} y_{1} y_{2}+p_{2}^{2}\left(y_{2}^{2}-2\right)+4\right)+4 q_{2} \sqrt{-p_{2}^{2}-q_{2}^{2}+2}\left(p_{1}^{2} y_{1}+p_{2}^{2} y_{2}\right) \\
& +\left(p_{1}^{2} y_{1}+p_{2}^{2} y_{2}\right)^{2}+4 q_{1}^{3} y_{1} \sqrt{-p_{1}^{2}-q_{1}^{2}+2} \\
& \left.+4 q_{2}^{3} y_{2} \sqrt{-p_{2}^{2}-q_{2}^{2}+2}+q_{1}^{4}\left(y_{1}^{2}-4\right)+q_{2}^{4}\left(y_{2}^{2}-4\right)\right),
\end{aligned}
$$

which is defined in a four-dimensional phase space, though energy conservation allows to reduce it to three dimensions only. The dynamics of the system is determined by Hamilton's equations:

$$
\begin{aligned}
\frac{d q_{k}}{d t} & =\frac{\partial h_{c l}}{\partial p_{k}} \\
\frac{d p_{k}}{d t} & =-\frac{\partial h_{c l}}{\partial q_{k}},
\end{aligned}
$$

with $k=1,2$. Once Eqs. (13) are solved numerically, the nature of the motion, either regular or chaotic, can be easily depicted using the Poincaré sections, plotting the coordinate values of the intersection in a given plane of different trayectories of the system. In our case, we consider intersections with the plane $p_{2}=0$ (note that $q_{2}$ is determined by energy conservation), and then we collect the coordinates $q_{1}-p_{1}$. On the other hand, chaotic regimes lead to trayectories that occupy the whole available phase space and intersect randomly the Poincaré section. On the other hand, regular situations correspond to trajectories that are constrained to toroidal regions that generate closed curves when they intersect the Poincaré section. 
Finally, another very simple tool that is able to distinguish qualitatively regular from chaotic states is the Peres lattice [33]. A Peres lattice provides a way to characterize states by simply performing a diagram where each point corresponds to a single state. In the diagram the matrix element of a convenient operator is plotted versus the excitation energy. In our case, an efficient operator is $n_{t_{i}}$. Therefore, we will represent $\left\langle n_{t_{1}}\right\rangle / N_{1}$ (or $\left.\left\langle n_{t_{2}}\right\rangle / N_{2}\right)$, whose values range between 0 and 1 , as a function of the excitation energy. In the case of an energy region with a chaotic behaviour, the Peres lattice provides a disordered distribution of points, while for regular states, the pattern becomes ordered. Even in the case of coexistence of regular and chaotic states in the same energy region, a Peres lattice will allow to separate both families. Note that the Peres lattice can also help to define the "shape/phase" of the excited states, as explained in [23]. The characterization of the excited states "shape/phase" is carried out by examination of the pattern observed in the wave functions within a region, but not for a single state. Peres lattices are an ideal tool to characterize "shape/phase" excited states within a given region.

\section{Participation ratio}

A different way of studying the onset of an ESQPT is through the analysis of the wave function. In particular, it is enlightening to study how localized or delocalized is a given state since it has been recently proven [34] that states nearby the critical energy of an ESQPT are well localized, while the rest present sparse wave functions. A convenient quantity to study the structure of the wave function is the participation ratio, $P$, which is defined, in a given basis $\{|i\rangle\}$, for a wavefunction $\left|\psi_{k}\right\rangle=\sum_{i} C_{i}^{(k)}|i\rangle$ as,

$$
P^{(k)}=\frac{1}{\operatorname{dim}} \frac{1}{\sum_{i}\left|C_{i}^{k}\right|^{4}},
$$

where dim stands for the dimension of the Hilbert space. This function provides the degree of delocalization of a given state in a particular basis, being, therefore, basis dependent. For a well localized state, $P$ is small (1/dim in value), while it will become large for a delocalized one (1 as maximum value).

In Ref. [34], the authors showed that using a $u(n)$ basis, the participation ratio of the nearest eigenstate to the ESQPT shows a marked dip that allows to localize very cleanly the ESQPT position. In this work we will use the $u_{1}(1) \otimes u_{2}(1)$ basis in which the quantum 
numbers $n_{t_{1}}$ and $n_{t_{2}}$ are specified, as explained in Ref. [21].

\section{CASES OF INTEREST}

The first case we deal with is $y=0, y^{\prime}=0\left(y_{1}=0, y_{2}=0\right)$, this is the line from $u_{12}(1)$ to $s o_{12}(2)$ ), with $x=1 / 2$, which corresponds to a deformed phase after the critical point of a second order QPT at $x_{c}=4 / 5$. This case is represented with point A in Fig. 1 . In the following, all the calculations will be performed for $N_{1}=N_{2}=70$ bosons, which involves a dimension of $71 \times 71=5041$. This Hamiltonian generates a deformed phase for the ground state, i.e., the order parameters are $\beta_{1} \neq 0$ and $\beta_{2} \neq 0$. Because $y_{1}=y_{2}$ the system is symmetric under the interchange of the index 1 and 2 . Therefore, $u_{12}(2)$ will be the dynamical algebra of the Hamiltonian and the states will belong to a certain Young tableau, $\left[h, h^{\prime}\right]$, making possible to define an angular momentum quantum number $j=1 / 2\left(h-h^{\prime}\right)$ with possible values $j=1 / 2\left(N_{1}+N_{2}\right), 1 / 2\left(N_{1}+N_{2}\right)-1, \ldots, 1 / 2\left|N_{1}-N_{2}\right|$, as shown in Sec. II. As a consequence, the Hilbert space can be split in subspaces with a given value of $j$ and $2 j+1$ dimension. This fact should be taken into account for interpreting correctly the coming figures, otherwise wrong conclusions can be reached.

Let us start with the study of the density of states (Fig. 33), for which we already saw in Fig. 2 how a lambda divergence is obtained at the critical energy of the ESQPT. In Fig. 3a, the value of the density of states for selected values of $j$ is depicted (we use a different color for each $j$ value), for each of them a peak is clearly observed, which is a precursor of a lambda divergence. Note also that as $j$ decreases the energy of each peak is slightly shifted to higher values of the energy. In panel (b), the density corresponds to the sum of the individual densities presented in panel (a) and the consequence is that the typical density values in panel (b) are much larger than in panel (a) and that individual peaks no longer can be observed and, indeed, the only observed peak is a broad one well above zero energy. The energy of the peak at $E \approx 30$ is connected with the peak position of the families with the smallest $j$ values, whose peaks are located at the highest energy values. Consequently, for this case the level density is not a good marker for the ESQPT.

In Fig. 4 we plot the Peres lattice for $\frac{\left\langle n_{t_{1}}\right\rangle}{N_{1}}$ as a function of the excitation energy: in panel (a) the plot is done for each $j$ (different color for each $j$ ) while in panel (b) all the states (all $\left.j^{\prime} s\right)$ are considered together. In the latter case one can observe an almost global regularity, 

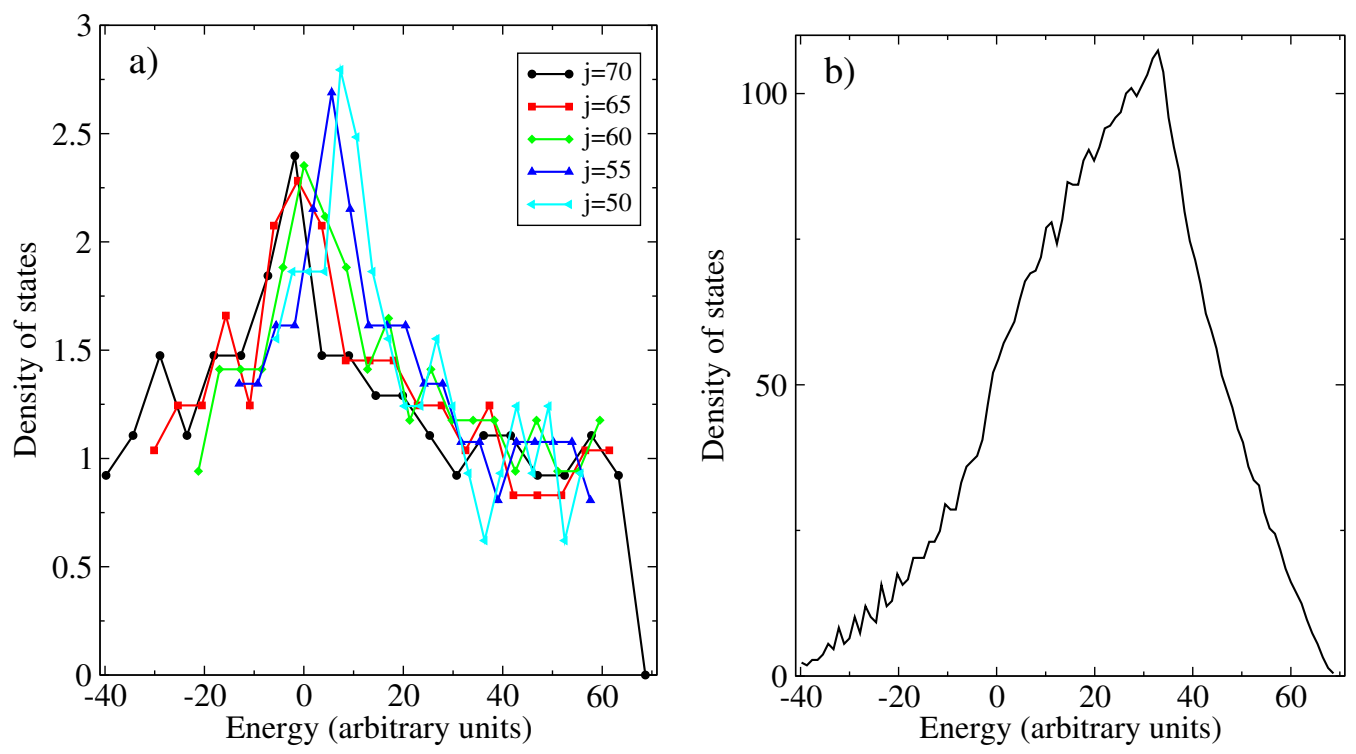

FIG. 3: Density of states as a function of the excitation energy for a Hamiltonian with parameters $y=0, y^{\prime}=0$, and $x=1 / 2$ and a number of bosons $N_{1}=N_{2}=70$. a) For selected values of $j, \mathrm{~b}$ ) for all the states without distinction in $j$.

but taking into account what is observed in panel (a), one can notice that such a regularity is associated with the presence of $j$ as an extra quantum number. The main feature of each set of points, corresponding to given $j$-values, is the sharp dip observed at energies around $E \approx 0$, with a shift in the dip position towards higher energies as $j$ decreases, in a similar way to Fig. 3a. Therefore, Fig. 4b is simply the superposition of different curves with sharp dips that move from $E \approx 0$ to $E \approx 30$. Both below and above the ESQPT, the Peres lattice shows an ordered pattern, pointing to a non-chaotic behaviour. Note that in this case, we do not analyze the NNSD because we have to separate in sets with the same value of $j$ and, therefore, the number of states will be too low to calculate a reliable value of $\eta$ (8). However, one can calculate the Poincaré sections to study the regularity and chaos interplay. In panels (c), (d), (e), and (f) of Fig. 4 we depict the Poincaré sections for energies $E=-20, E=0$, $E=30$, and $E=50$, respectively. Because of the underlying $U_{12}(2)$ symmetry, all the cases correspond to a regular regime.

In Fig. 5 we plot the value of the participation ratio as a function of the excitation energy. Once more, in panel (a) we separate in families with given $j$-values (different colors) and in panel (b) we plot all the states. In each family depicted in panel (a) one can see how the participation ratio starts increasing and reaches a maximum, then decreases with a 

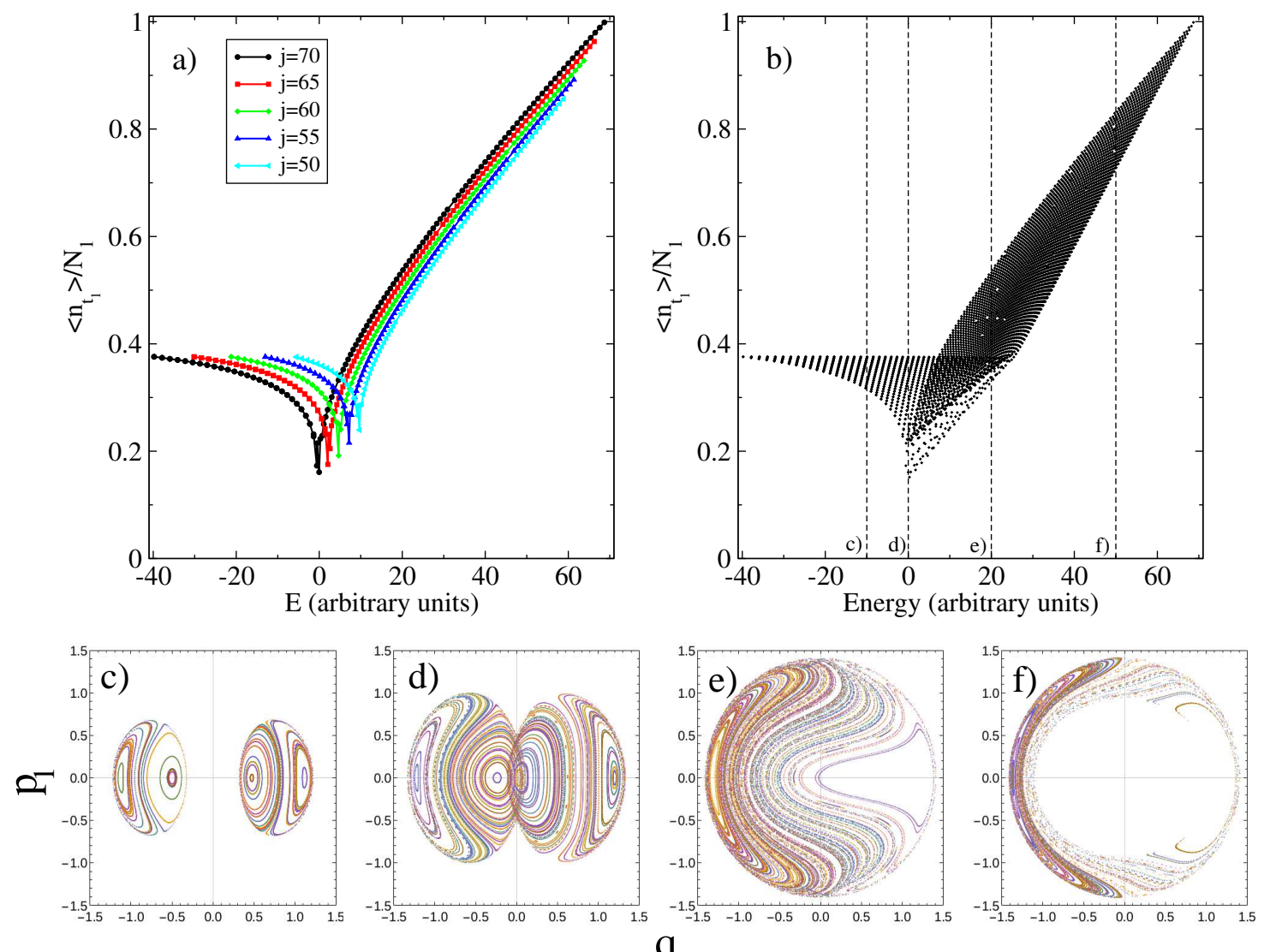

$\mathrm{q}_{1}$

FIG. 4: Peres lattice for $\frac{\left\langle n_{t_{1}}\right\rangle}{N_{1}}$ versus the excitation energy for the same Hamiltonian and parameters as Fig. 3. a) For selected values of $j$, b) for all the states without distinction in $j$. Poincaré sections for energies $E=-10, E=0, E=20$, and $E=50$, in panels c), d), e), and f), respectively (energies marked in panel (b)).

sharp minimum, then shows a new maximum, and finally ends with a minimum. This behaviour was already described in [34] and simply reflects the strong "localization" of the wave function at the ESQPT. Once more, the position of the minimum moves towards higher energies for decreasing $j$-values as it is shown in Figs. 3 and 4 . Panel (b) corresponds to the superposition of the previously described curves which leads to the presence of certain parabolic curves somehow blurred. The set of well defined lines in the right bottom part of Fig. 5b corresponds to curves with small values of $j$.

The second selected case of interest corresponds to parameters $y=1, y^{\prime}=0\left(y_{1}=1\right.$, $\left.y_{2}=1\right)$, this is a line in the base of the phase diagram going from $u_{12}(1)$ to the deformed region crossing the line of first order phase transition which is located at $x_{c} \approx 4 / 5$ (point B 

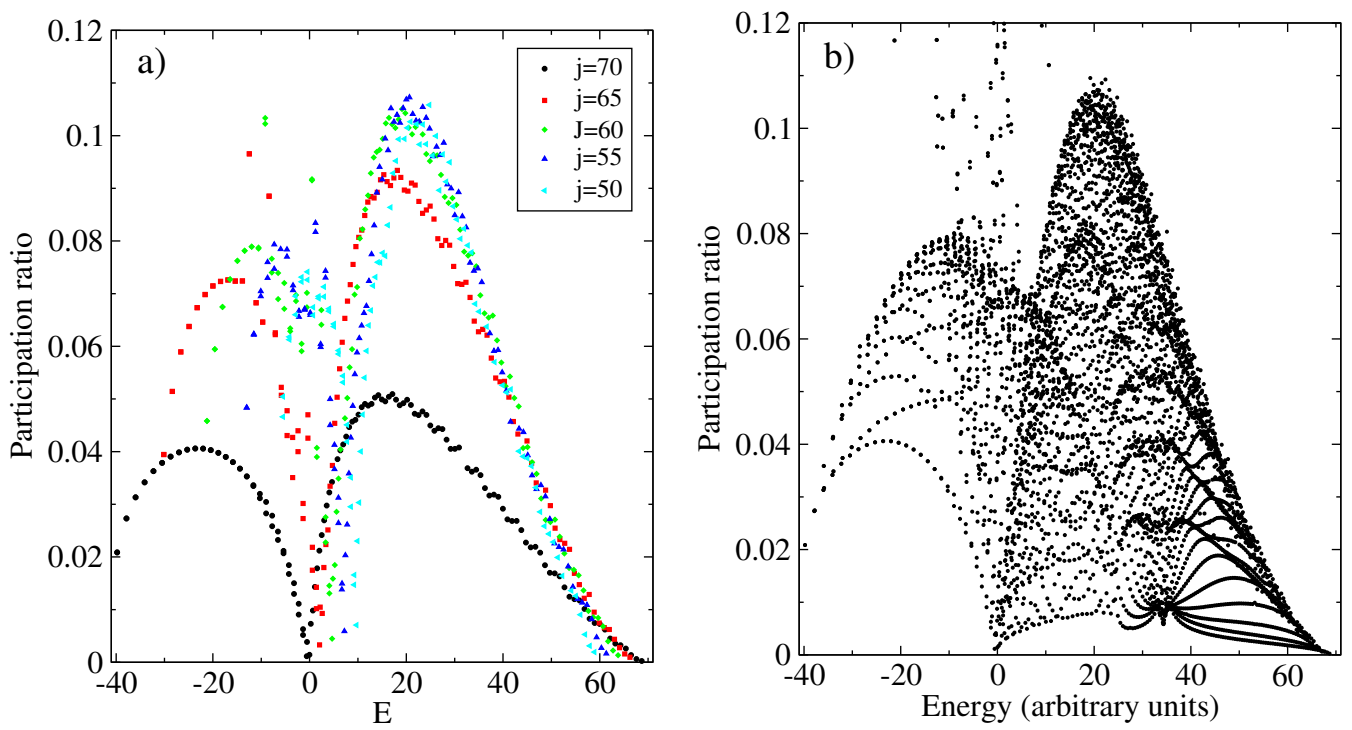

FIG. 5: Normalized participation ratio as a function of the excitation energy for the same Hamiltonian and parameters as in Fig. 3. a) For selected values of $j$, b) for all the states without distinction in $j$.

in Fig. 1). In particular we have selected the point $x=1 / 2$. For this selection of the control parameters, the system ground state is deformed and since $N_{1}=N_{2}$, the indexes 1 and 2 can be interchanged, $u_{12}(2)$ is the dynamical algebra of the Hamiltonian and $j$ is a good quantum number. Therefore, we are in a similar situation to the case previously discussed but now, besides the ESQPT, a set of states associated with the second (local) minimum of the potential energy surface will appear.

We start with the analysis of the density of states. In Fig. 6, the density of states are plotted versus the excitation energy. In panel (a) each color line corresponds to a given $j$-value, while in panel (b) the total density of states is plotted independently of the $j$-values. In panel (a), in addition to the peak close to zero that is the precursor of the lambda divergence, a previous finite discontinuity is observed very close in energy (negative). In panel (b), a relatively broad peak is observed above zero energy, but nothing qualitatively different to Fig. 3b which corresponds to an ESQPT without a local minimum in the potential energy surface.

In Fig. 7 we present the Peres lattice for $\frac{\left\langle n_{t_{1}}\right\rangle}{N_{1}}$ versus the excitation energy for given $j$-values (different colors) in panel (a), or for the whole set of states in panel (b). Here, narrow dips are observed in panel (a) around the energy of the ESQPT. The main difference 

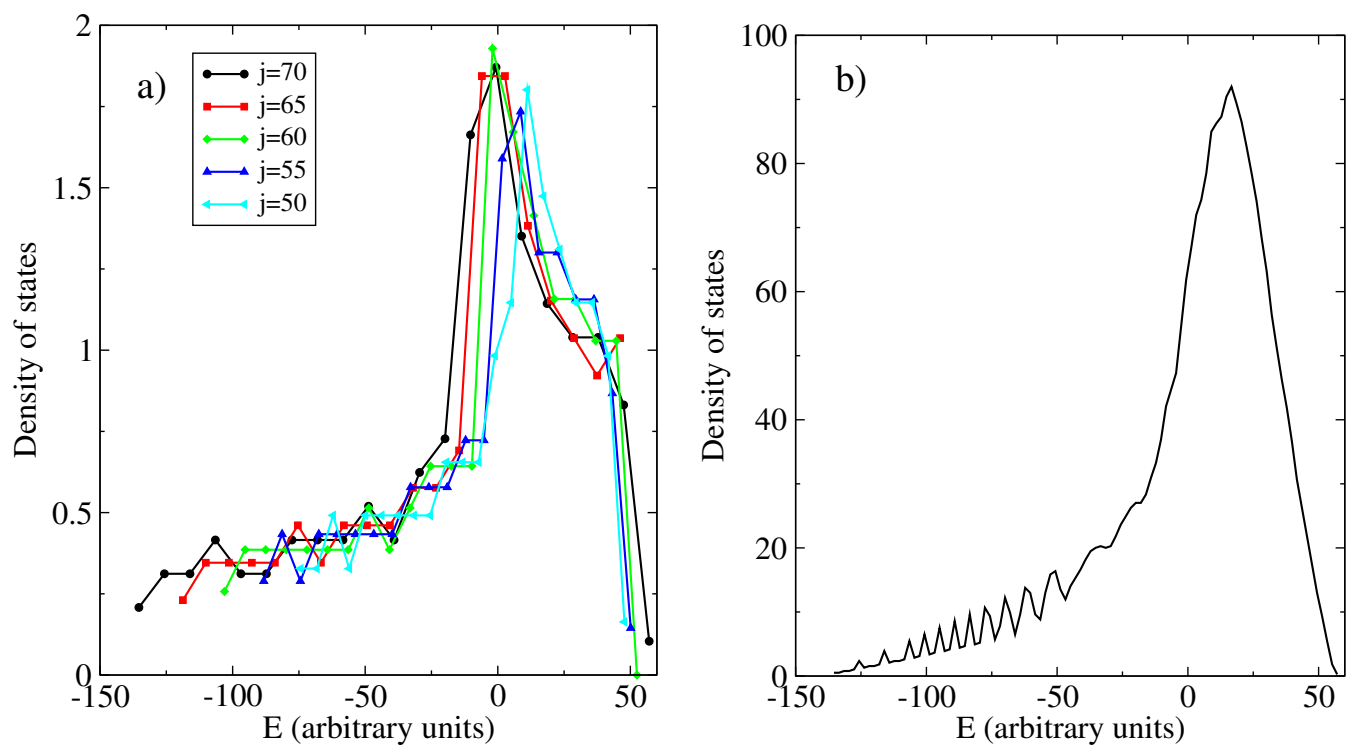

FIG. 6: Density of states as a function of the excitation energy for a Hamiltonian with parameters $y=1, y^{\prime}=0$, and $x=1 / 2$ and a number of bosons $N_{1}=N_{2}=70$. a) For selected values of $j, \mathrm{~b}$ ) for all the states without distinction in $j$.

with respect to the previous case is the appearance of a second family of states (see lowest part of Fig. 7 in both panels) related with the local minimum of the potential energy surface. Note that also in this case, we do not analyze the NNSD because we have to separate in sets with the same $j$-value and, therefore, the states will be too few to calculate the value of $\eta$. As in previous case, one can calculate the Poincaré sections to study the regularity and chaos interplay. In panels (c), (d), (e), and (f) of Fig. 7 we depict the Poincaré sections for energies $E=-10, E=0, E=20$, and $E=50$, respectively. Because of the underlying $U_{12}(2)$ symmetry, all the cases correspond to a regular regime, too.

In Fig. 8 we depict the participation ratio value as a function of the excitation energy, for selected particular $j$-values (different colors) in panel (a), and for the whole set of states in panel (b). In panel (a) each family of states has a narrow minimum around zero energy with broad maxima at right and left. Here, for each $j$-family a second set of states, related with the local maximum of the energy surface, appears just below the energy of the ESQPT, but it can be almost unnoticed. It is worth to mention that even in panel (b) the position of the ESQPT critical energy is clearly marked with a relatively narrow dip.

Till now we have only studied cases where $u_{12}(2)$ was the dynamical algebra of the Hamiltonian and, therefore, they essentially correspond to the superposition of several one- 

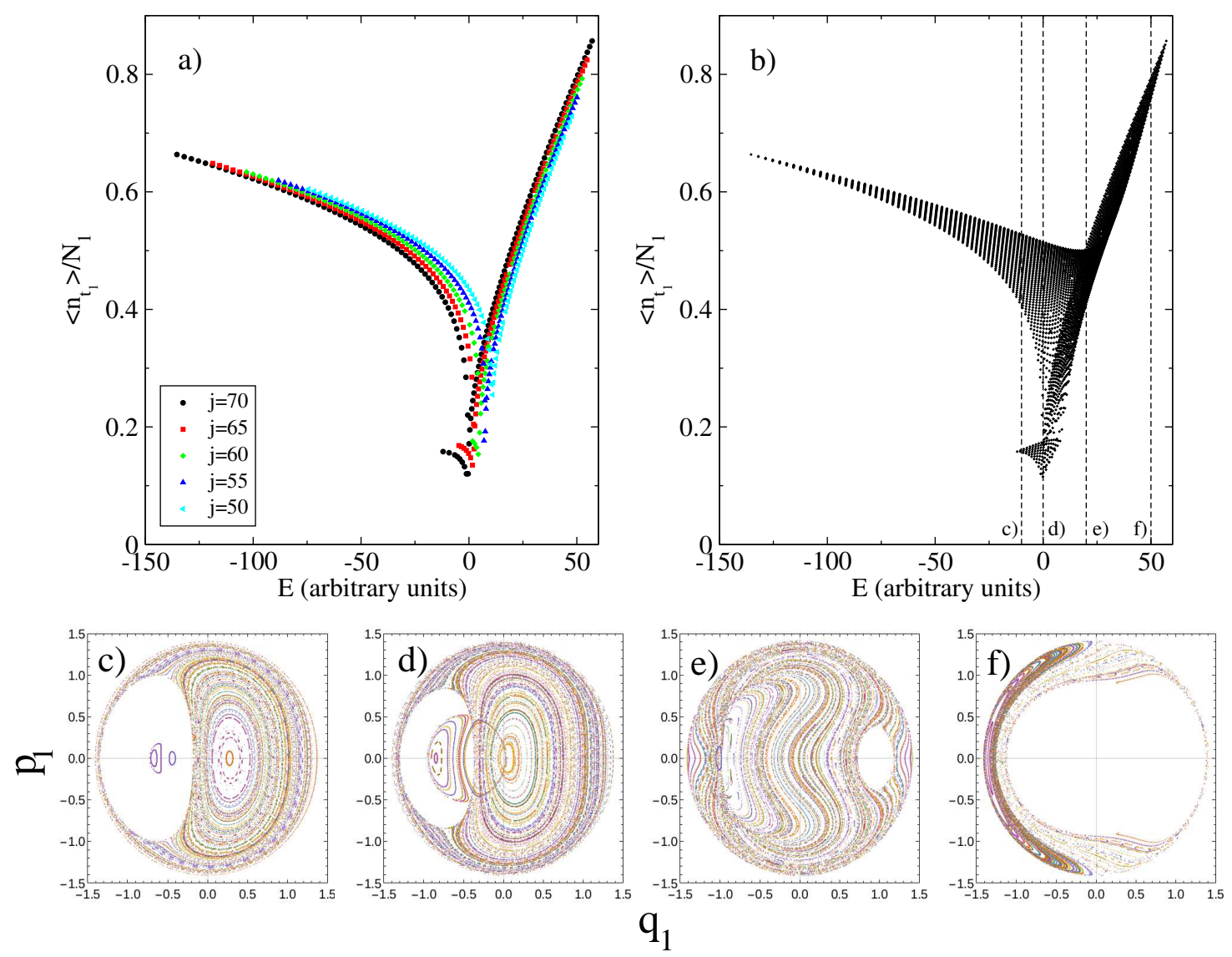

FIG. 7: Peres lattice for $\frac{\left\langle n_{1}\right\rangle}{N_{1}}$ versus the excitation energy for the same Hamiltonian and parameters than in Fig. 6. a) For selected values of $j$, b) for all the states without distinction in $j$. Poincaré sections for energies $E=-10, E=0, E=20$, and $E=50$, in panels c), d), e), and f), respectively (energies marked in panel (b)).

fluid Lipkin systems with $2 j$ number of bosons. Now we will move to the more interesting $y_{1} \neq y_{2}$ cases, where $j$ is no longer a good quantum number. The already studied cases have taught us how the patterns observed in Figs. 3b, 4b, 5b, 6b, 7b, and $8 \mathrm{~b}$ are a consequence of the superposition of lines corresponding to different $j$-values, in other words, a consequence of the underlying $u_{12}(2)$ symmetry.

Next case of interest corresponds to the parameter values $y=1 / 4, y^{\prime}=3 / 4\left(y_{1}=1\right.$, $\left.y_{2}=-1 / 2\right)$ which is a point in the line from spherical $u_{12}(1)$ to the deformed region but not in the base of the phase diagram, and consequently does not preserve the $u_{12}(2)$ symmetry. This line crosses the first order surface located around $x_{c} \approx 4 / 5$. We have selected the point $x=1 / 2$ which corresponds to a deformed ground state (point $\mathrm{C}$ in Fig. 1). In this case, as 

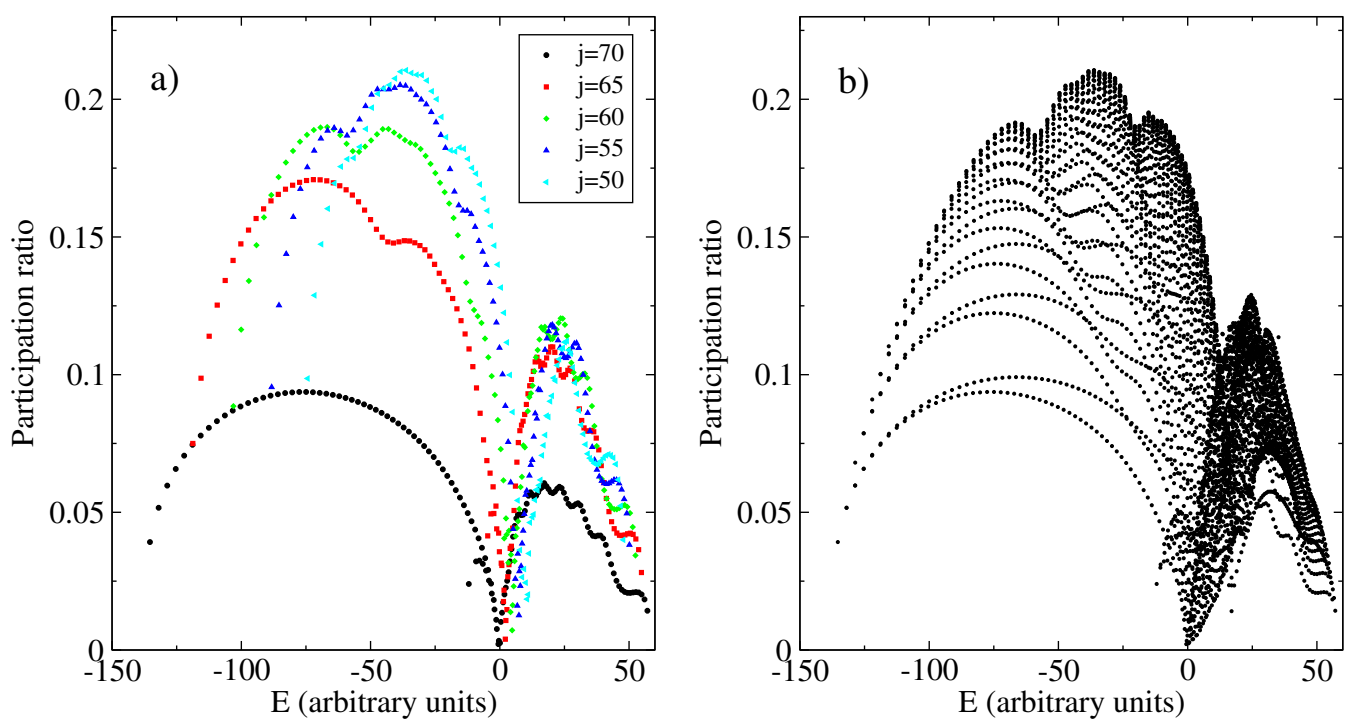

FIG. 8: Participation ratio as a function of the excitation energy for the same Hamiltonian and parameters as in Fig. 6. a) For selected $j$-values, b) for all the states without distinction in $j$.

already told, it is no longer possible to separate the states in terms of $j$. The potential energy surface of this Hamiltonian has a global deformed minimum and another local deformed one separated by a maximum. In Fig. 9a, the density of states is plotted as a function of the excitation energy. It can be observed that this quantity is not marking correctly the presence of the ESQPT at zero energy, as already learnt from the preceding calculations. It is worth noting that, although this case presents a second family of states associated to the local minimum starting at $E \approx-40$, nothing is observed in the density of states. In Fig. $9 \mathrm{~b}$ the Peres lattice for $\left\langle n_{t_{1}}\right\rangle / N_{1}$ is depicted as a function of the excitation energy. This quantity shows three clear regions, two on them at energies below the ESQPT critical energy (zero energy) and the third one above. The two lowest families of points correspond to states that are located in the well of the global deformed minimum $\left(\left\langle n_{t_{1}}\right\rangle / N_{1} \approx 0.6\right)$ and in the well of the local one $\left(\left\langle n_{t_{1}}\right\rangle / N_{1} \approx 0.2\right)$, also deformed. These two regions show a very regular pattern. The third region is above $E \approx 0$ and presents a relatively disordered structure, except in particular regions, as is $0.8<\left\langle n_{t_{1}}\right\rangle / N_{1}<1$. The Peres lattice is clearly showing the existence of two different deformed phases, non-symmetric, with a more regular behaviour below zero energy and a symmetric phase with certain degree of chaoticity above the energy of the ESQPT. In panel (b) the value of $\eta$ (red line), representing the NNSD, as a function of the energy is plotted too. This quantity shows a sudden decrease at the energy of the ESQPT, therefore pointing to a spectrum with a more regular behaviour 
below the ESQPT and more chaotic above. However, even above the ESQPT, there is a regular region at $E \approx 50$ with $\left\langle n_{t_{1}}\right\rangle / N_{1} \approx 0.7$. This behaviour has been already observed in other models such as the Bose-Hubbard Hamiltonian [35]. Note that we do not reach the whole range of energies because we exclude the $10 \%$ of states with lowest and highest energies to calculate $\eta$. In Fig. 9c it is depicted the participation ratio versus the excitation energy. Here, one can note a rather different structure for energies below and above the critical energy of the ESQPT. For energies below zero, one can see two sets of inverted parabolas, with a minimum around zero. The two sets correspond to the two branches already seen in Fig. 9b. For energies above the ESQPT a single inverted parabola, though rather blurred, is observed. This region presents a behaviour similar to the one obtained with $u_{12}(2)$ conserving Hamiltonians. Finally, panels (d), (e), (f) and (g) correspond to Poincaré sections with energies $E=-20, E=0, E=30$, and $E=50$, respectively. These figures confirm the latter statements, i.e., that below the ESQPT energy a regular behaviour exits, as panel (d) confirms, at the ESQPT energy chaotic orbits star to appear (as shown in panel (e)), and above the ESQPT energy, regions with chaotic (see panel (f)) and partially regular motion coexist (see panel $(\mathrm{g})$ ).

The three last cases correspond to Hamiltonians with $y=0$, i.e., with $y_{1}=-y_{2}$, and, therefore, located on the gray vertical plane of the phase diagram. As we proved in [21], for values of $y^{\prime}<1$ a second order QPT appears at $x_{c}=4 / 5$, for $y^{\prime}=1$ the QPT shows a divergence in $d^{2} E / d x^{2}$ also at $x=4 / 5$, but for $y^{\prime}>1$ the QPT becomes of first order. Thus, next we try to disentangle whether or not there is a different qualitative behaviour between these three situations in terms of analyses of density of states, Peres lattice, Poincaré section, and participation ratio. In Fig. 10 the results for the case of $y^{\prime}=1 / 2$ are presented, in Fig. 11 the case of $y^{\prime}=1$ is studied, and, finally, in Fig. 12 the case of $y^{\prime}=3 / 2$ is analyzed.

In Fig. 10 we consider the case $y=0, y^{\prime}=1 / 2$ and $x=1 / 2$ (point D in Fig. 1). This corresponds to a deformed ground state in a line that changing $x$ presents a second order QPT at $x_{c}=4 / 5$. The energy surface has two deformed degenerate minima (at $\beta$ and $-\beta$ ) separated by a spherical maximum. In panel (a) the value of the density of states is presented as a function of the excitation energy. A relatively broad peak is observed with a maximum well above the energy of the ESQPT. In panel (b) the Peres lattice for $\left\langle n_{t_{1}}\right\rangle / N_{1}$ as a function of excitation energy is presented. Note that because of the presence of degenerated doublets, it is needed to include a tiny perturbation in the Hamiltonian for slightly break 

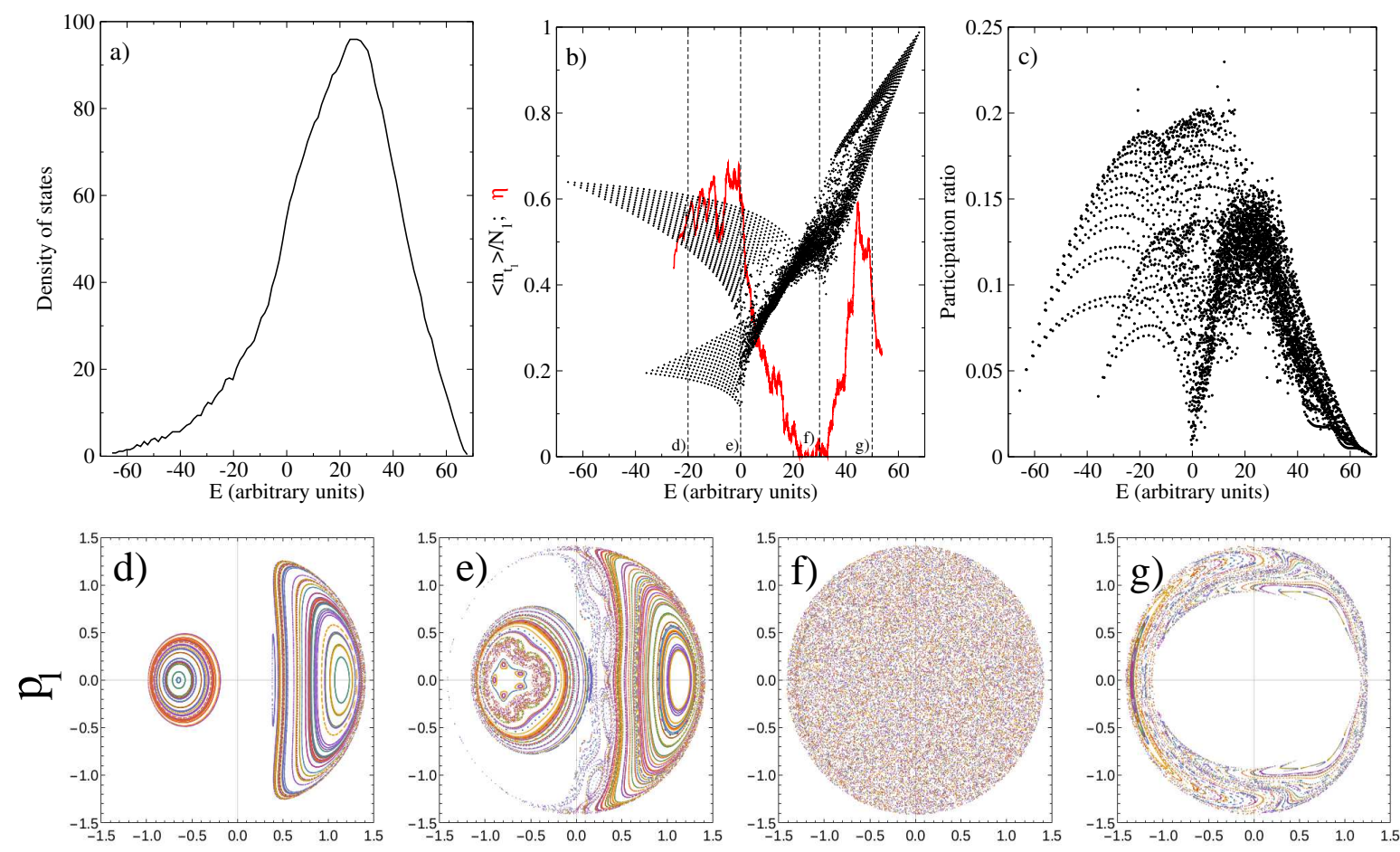

$\mathrm{q}_{1}$

FIG. 9: Different observables as a function of the excitation energy for a Hamiltonian with parameters $y=1 / 4, y^{\prime}=3 / 4$, and $x=1 / 2$ and a number of bosons $N_{1}=N_{2}=70$ : a) Density of states, b) Peres lattice (black points) for $\frac{\left\langle n_{t_{1}}\right\rangle}{N_{1}}$ and $\eta$-value (red solid curve), c) participation ratio, d), e), f), and g) Poincaré sections for energies $E=-20, E=0, E=30$, and $E=50$, respectively (energies marked in panel (b)).

the degeneracy and avoid random linear combinations of states. Two branches of points are clearly seen for energies below the ESQPT, one centered around $\left\langle n_{t_{1}}\right\rangle / N_{1} \approx 0.3$ and the other around $\left\langle n_{t_{1}}\right\rangle / N_{1} \approx 0.5$. These two branches correspond to the two degenerated and symmetric minima for which $\left\langle n_{t_{1}}\right\rangle$ and $\left\langle n_{t_{2}}\right\rangle$ are interchanged. In both cases a clear ordered pattern is observed. Note that for each energy there exist two degenerated points, one in each branch. Above the ESQPT the order is lost and the points are located in a more or less random way around a straight line. As in previous cases, the ESQPT separates the regular and the chaotic zones. In this sense, the ESQPT separates two "shapes/phases" of the system. This is confirmed through the calculation of the NNSD $\eta$-value, which shows a sudden decrease in its value at zero energy, though with several oscillations, therefore having a more regular behaviour below the ESQPT and more chaotic above. However, even above 

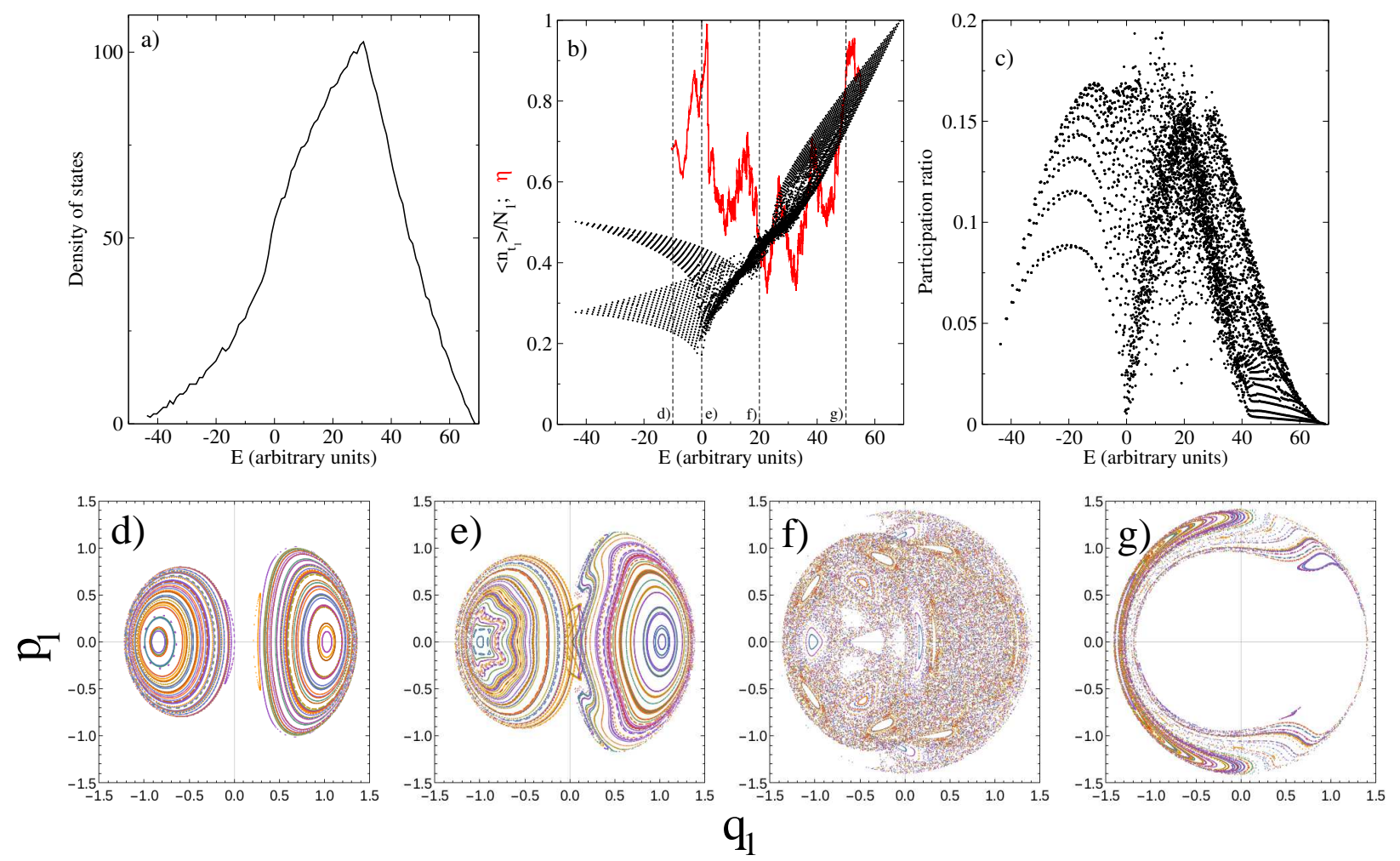

FIG. 10: Same as in Fig. 9 but for $y=0, y^{\prime}=1 / 2$, and $x=1 / 2$. Panels d), e), f), and g) are Poincaré sections for energies $E=-10, E=0, E=20$, and $E=50$.

the ESQPT, there is a more regular region at $E \approx 50$ with $\left\langle n_{t_{1}}\right\rangle / N_{1} \approx 0.8$. Panels (d), (e), (f) and (g), which correspond to Poincaré sections with energies $E=-10, E=0, E=20$, and $E=50$, respectively, confirm the above findings: below the ESQPT energy a regular behaviour exits (panel (d)), at the ESQPT energy chaotic orbits star to appear (as shown in panel (e)), and above the ESQPT energy, regions with chaotic (panel (f)) and partially regular motion coexist (see panel $(\mathrm{g})$ ). In panel (c) it is depicted the participation ratio versus the excitation energy. Below the ESQPT, the points (doubly degenerated) describe well separated inverted parabolas with a minimum at zero energy. For energies above the ESQPT, once more, the points define a very blurred parabola.

Next case to be analyzed is $y^{\prime}=1$ which is probably the most exotic one because the associated QPT at $x_{c}=4 / 5$ presents a divergence in $d^{2} E / d x^{2}$ (point E in Fig. 1). However, as we will explain, in fact, nothing special occurs in the spectrum. In Fig. 11a we depict the density of states and it looks like the one presented in Fig. 10a. In Fig. 11b we present the Peres lattice and, once more, we have two branches for energies below the ESQPT, one 

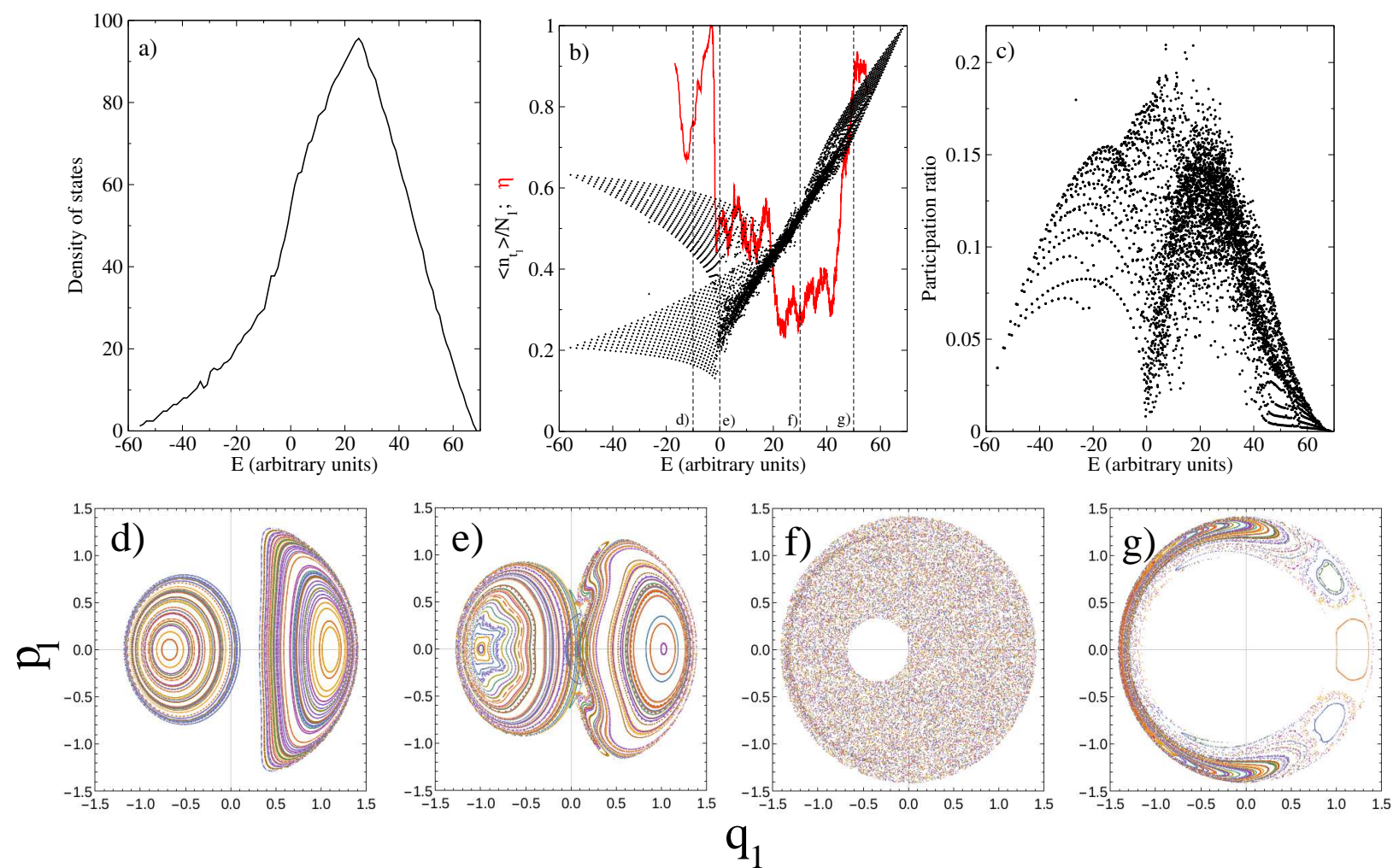

FIG. 11: Same as in Fig. 9 but for $y=0, y^{\prime}=1$, and $x=1 / 2$. Panels d), e), f), and g) are Poincaré sections for energies $E=-10, E=0, E=30$, and $E=50$.

centered around $\left\langle n_{t_{1}}\right\rangle / N_{1} \approx 0.2$ and the other around $\left\langle n_{t_{1}}\right\rangle / N_{1} \approx 0.6$. Note that for each energy there exist two degenerated points. Above the ESQPT we obtain a cloud of points scattered around a straight line. Here too, the NNSD $\eta$-value is plotted and once more it presents a sudden lowering at the ESQPT energy, therefore pointing to a more regular spectrum below the ESQPT, while more chaotic above. Note that to calculate $\eta$ below the energy of the ESQPT, we only consider states in one of the branches, otherwise the results are wrong. The decreasing occurs in two steps, the first at zero energy and the second at $E \approx 20$. This fact is a consequence of the coexistence of intruder and regular states in the same energy region. In both regions several oscillations are observed. Finally, there is an striking increase of $\eta$ at $E \approx 50$, pointing to the presence of a regular region above the ESQPT, as can be also observed in the Peres lattice. Once more, panels (d), (e), (f) and (g), which correspond to Poincaré sections with energies $E=-10, E=0$, $E=30$, and $E=50$, respectively, confirm the above findings: below the ESQPT energy a regular behaviour exits (panel (d)), at the ESQPT energy chaotic orbits star to appear 

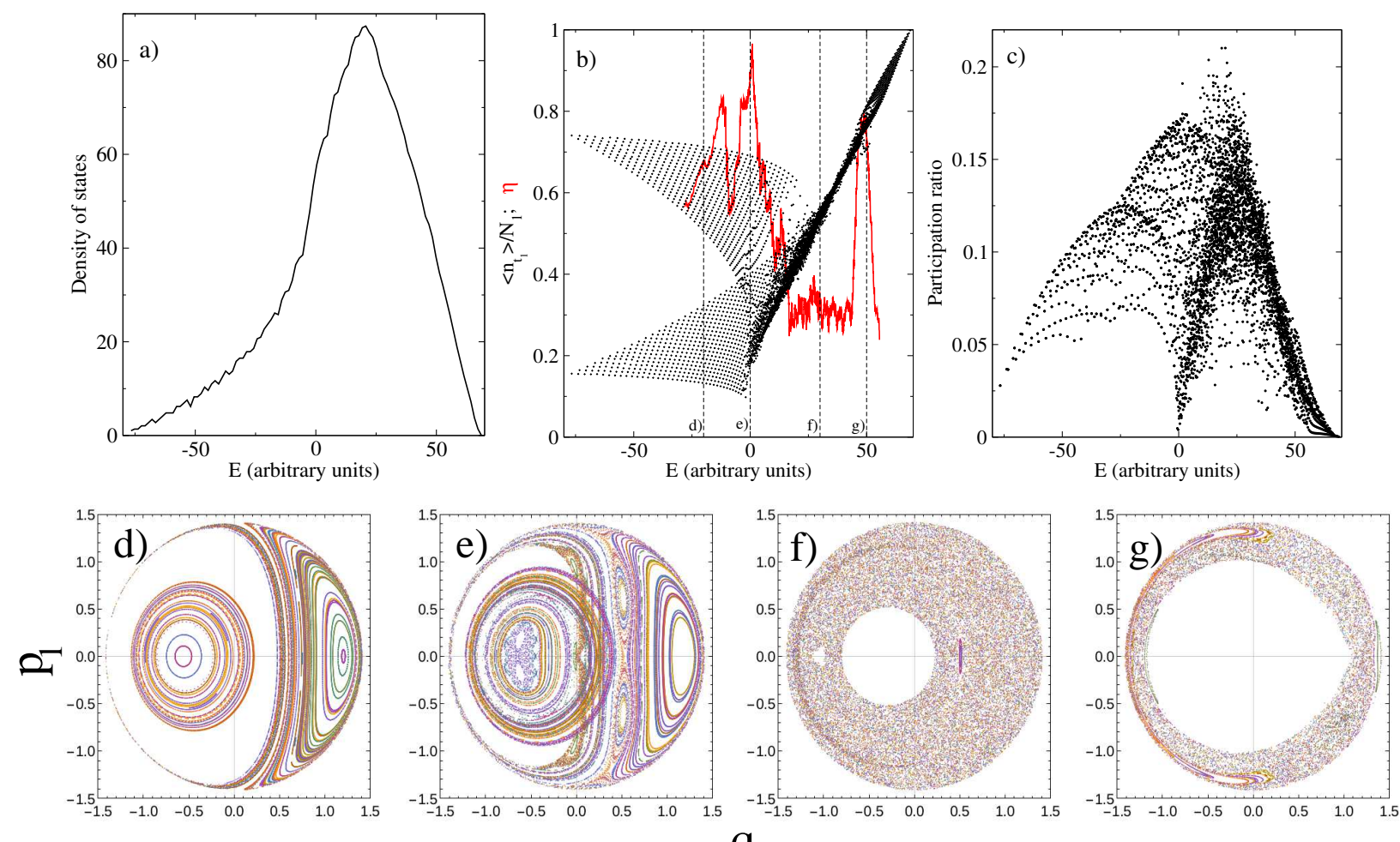

$\mathrm{q}_{1}$

FIG. 12: Same as Fig. 9 but for $y=0, y^{\prime}=3 / 2$, and $x=1 / 2$. Panels d), e), f), and g) are Poincaré sections for energies $E=-20, E=0, E=30$, and $E=50$.

(as shown in panel (e)), and above the ESQPT energy, regions with chaotic (panel (f)) and partially regular motion coexist (see panel (g)). In Fig. 11k the participation ratio is plotted, presenting well separated inverted parabolas below the ESQPT, with the minimum at zero energy, while above the ESQPT a single thick inverted parabola can be defined.

The latest case to be analyzed is $y=0, y^{\prime}=3 / 2$ which is a line that presents a first order QPT for $x_{c} \approx 4 / 5$. The value $x=1 / 2$ corresponds to an energy surface with two degenerate deformed minima (point $\mathrm{F}$ in Fig. 1), as in previous cases. The obtained figures, 12 a for the density of states, $12 \mathrm{~b}$ for the Peres lattice and, $12 \mathrm{c}$ for the participation ratio, present only slightly differences with respect to Fig. 11. In particular, in the Peres lattice, the two branches located below the ESQPT are centered around $\left\langle n_{t_{1}}\right\rangle / N_{1} \approx 0.15$ and $\left\langle n_{t_{1}}\right\rangle / N_{1} \approx$ 0.75, respectively. Here, the $\eta$-value changes rapidly when crossing the ESQPT, separating the regular region below the ESQPT and the chaotic one above. Once more, around $E \approx 50$ there is a revival of the value of $\eta$ because of the occurrence of a more regular region. The rest of features are qualitatively the same as in Figs. 10 and 11, including participation ratio 
and Poincaré sections.
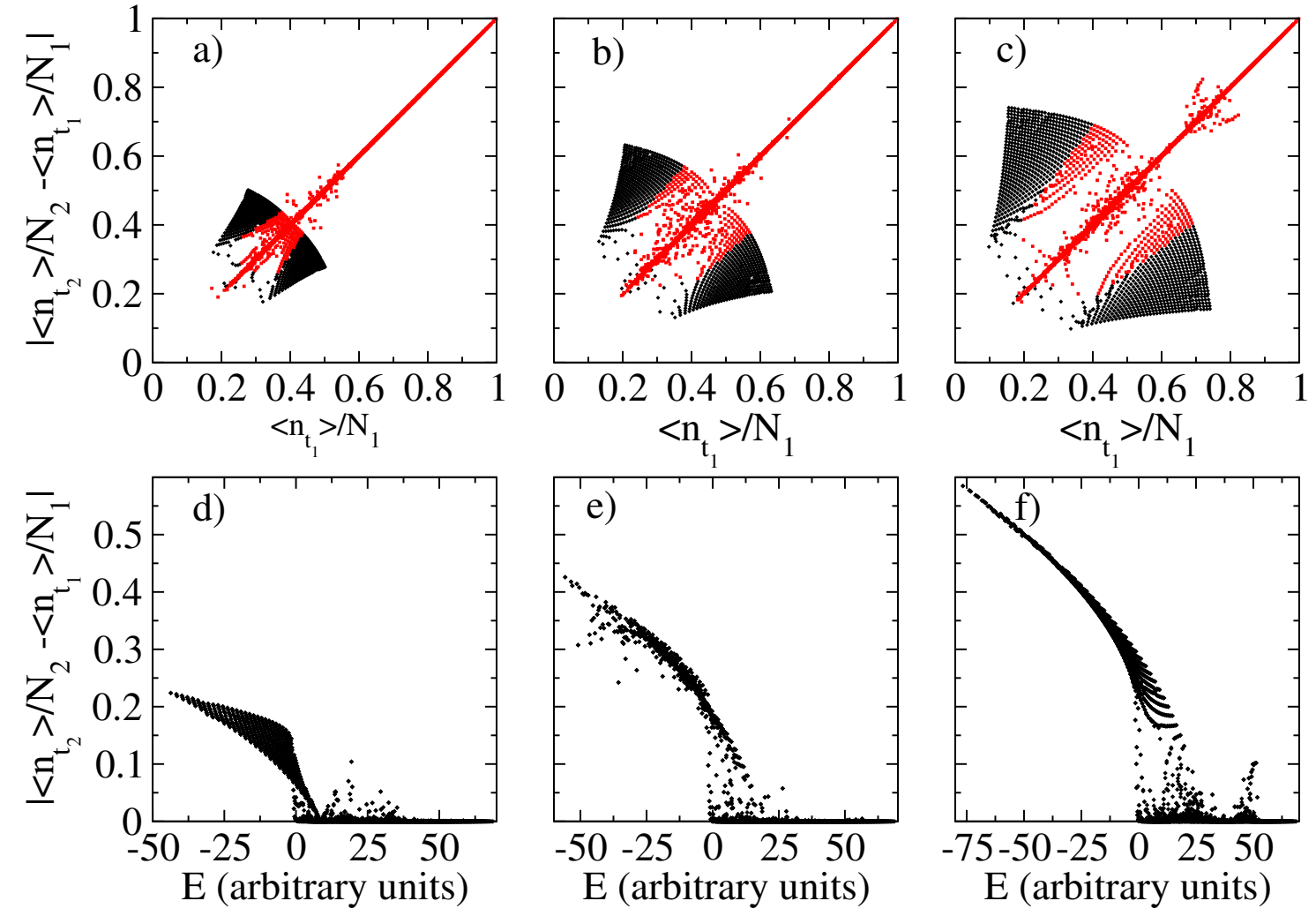

FIG. 13: $\left\langle n_{t_{1}}\right\rangle / N_{1}$ versus $\left\langle n_{t_{2}}\right\rangle / N_{2}$ for the Hamiltonians studied in Fig. 10 with parameters $x=1 / 2$, $y=0$, and $y^{\prime}=1 / 2\left(\right.$ panel (a)), in Fig. 11 with parameters $x=1 / 2, y=0$, and $y^{\prime}=1$ (panel (b)), and in Fig. 12 with parameters $x=1 / 2, y=0$, and $y^{\prime}=3 / 2$ (panel (c)). Black points correspond to states with negative energy, while the red ones correspond to states with positive energy. In panels (d), (e), and (f) we depict $\left\langle n_{t_{2}}\right\rangle / N_{2}-\left\langle n_{t_{1}}\right\rangle / N_{1}$ as a function of the energy for the same parameters than in panels (a), (b), and (c), respectively.

An alternative way of seeing the existence of different phases in the spectrum is the use of a two-dimensional diagram representing $\left\langle n_{t_{1}}\right\rangle / N_{1}$ versus $\left\langle n_{t_{2}}\right\rangle / N_{2}$. Moreover, we will see that $\left|\left\langle n_{t_{1}}\right\rangle / N_{1}-\left\langle n_{t_{2}}\right\rangle / N_{2}\right|$ can be considered as an order parameter and therefore will be a useful tool to define the phase of the system. We will consider the three last cases studied with $y=0$. In panels (a), (b), and (c) of Fig. 13 we depict $\left\langle n_{t_{1}}\right\rangle / N_{1}$ versus $\left\langle n_{t_{2}}\right\rangle / N_{2}$, which corresponds to $y^{\prime}=1 / 2, y^{\prime}=1$, and $y^{\prime}=3 / 2$, respectively. The black points correspond to states with negative energy, hence below the ESQPT, while the red ones correspond to states with positive energy, therefore above the ESQPT. In this figure one can easily single out the presence of two symmetric wells (they will become asymmetric if $y \neq 0$ ) 
that contain the black points, while the rest of states (red points) are scattered around the line $\left\langle n_{t_{1}}\right\rangle / N_{1}=\left\langle n_{t_{2}}\right\rangle / N_{2}$. Note that $\left\langle n_{t_{i}}\right\rangle / N_{i}$ can be connected with the order parameter

through $\beta_{i}=\sqrt{\frac{\left\langle n_{t_{i}}\right\rangle / N_{i}}{1-\left\langle n_{t_{i}}\right\rangle / N_{i}}}$. Panels (a), (b), and (c) of Fig. 13 provide a rough image of the available position space for the states below or above the ESQPT. For those below the ESQPT they are confined in two disjoint regions, where the potential in the Hamiltonian can be approximated by a quadratic form (one in each well) that leads to a regular regime [6, 29], while above the ESQPT, the harmonic approximation for the Hamiltonian is no longer valid and a more chaotic behaviour is expected. In panels (d), (e), and (f) we plot $\left|\left\langle n_{t_{2}}\right\rangle / N_{2}-\left\langle n_{t_{1}}\right\rangle / N_{1}\right|$ as a function of the energy, corresponding to $y^{\prime}=1 / 2, y^{\prime}=1$, and $y^{\prime}=3 / 2$, respectively. The variable $\left|\left\langle n_{t_{2}}\right\rangle / N_{2}-\left\langle n_{t_{1}}\right\rangle / N_{1}\right|$ has the typical behaviour of an order parameter, i.e., it has a zero value in the symmetric phase and different from zero in the non-symmetric one. In our case, states with an energy below the ESQPT owns a finite value of $\left|\left\langle n_{t_{2}}\right\rangle / N_{2}-\left\langle n_{t_{1}}\right\rangle / N_{1}\right|$ and it becomes zero for states with energy above the ESQPT.

\section{SUMMARY AND CONCLUSIONS}

In this work we have studied the onset of ESQPTs in a double Lipkin Hamiltonian which resembles the consistent-Q Hamiltonian of the interacting boson model. To find the presence of an ESQPT in the spectrum we relied on the study of the density of states, the Peres lattices, the Poincaré sections, the NNSD, and the participation ratio.

Taking into account the knowledge of the phase diagram of the model [21], we have selected particular points in the parameter model space that correspond to non-symmetric (deformed) phases and, therefore, should present an ESQPT in the spectrum at the energy at which the potential energy surface has a maximum, that in our case is always at zero energy. We have considered both cases with an ESQPT: the one with just one deformed minimum and a spherical maximum, and cases where two deformed minima appear separated by a spherical maximum. In this last case, a second family of states appears in the spectrum when reaching the excitation energy of the second minimum.

Among the analyzed cases, first we started with Hamiltonians with $u_{12}(2)$ as dynamical algebra and therefore with $j$ as good quantum number. We have learnt that when looking into the spectrum as a whole, one has to take into account that the symmetry of the Hamiltonian is shaping the results. This is particularly evident for the Peres lattice and 
the participation ratio. The conclusion for these cases is that the position of the ESQPT is shifted to higher energies as the value of $j$ decreases, but the main features of each ESQPT are the same already described in the literature.

Next we moved into cases where $u_{12}(2)$ symmetry was badly broken. Though the lack of symmetry, the patterns that we observed were amazingly similar to the previous cases and the zero energy clearly marks the edge between two shapes/phases with distinct patterns in the Peres lattice and in the participation ratio. The change in the structure of the Peres lattice, the Poincaré section, and in the NNSD $(\eta)$ points to the passing from a regular to a chaotic or less regular regime once the ESQPT is crossed, although above the ESQPT also appear regions with a strong regular character. The relationship between the appearance of an ESQPT and the onset of chaos, seems to depend on the Hamiltonian parameters, and therefore it is not possible to establish a clear connection between both phenomena, as pointed out in Ref. [32]. However, up to our knowledge, all the cases studied in the literature, including ours, point towards a regular behaviour for states below the ESQPT energy, i.e. belonging to the non-symmetric phase, although the character above the ESQPT energy strongly depends on the Hamiltonian. Finally, the use of $\left|\left\langle n_{t_{2}}\right\rangle / N_{2}-\left\langle n_{t_{1}}\right\rangle / N_{1}\right|$ as order parameter clearly mark the presence of an ESQPT at zero energy, which is deeply connected with the change in the observed pattern in the diagram $\left(\left\langle n_{t_{1}}\right\rangle / N_{1},\left\langle n_{t_{2}}\right\rangle / N_{2}\right)$ when crossing the ESQPT energy.

In summary, in a compound system, as the two-fluid Lipkin model, the existence of an ESQPT is self evident, though the value of the density of states turns out not to be an appropriated quantity to single out its presence. However, Peres lattices and participation ratio have shown to be ideal tools to mark the presence of ESQPTs.

\section{ACKNOWLEDGMENT}

This work has been supported by the Spanish Ministerio de Economía y Competitividad and the European regional development fund (FEDER) under Project No. FIS2014-53448- 
C2-1-P and FIS2014-53448-C2-2-P.

[1] R.F. Casten and E.A. McCutchan, Quantum phase transitions and structural evolution in nuclei, J. Phys. G 34, R285 (2007).

[2] P. Cejnar and J. Jolie, Quantum phase transitions in the interacting boson model, Prog. Part. Nucl. Phys. 62, 210 (2009).

[3] P. Cejnar, J. Jolie, and R.F. Casten, Quantum phase transitions in the shapes of atomic nuclei, Rev. Mod. Phys. 82, 2155 (2010).

[4] F. Iachello and F. Pérez-Bernal, Bending vibrational modes of ABBA molecules: algebraic approach and its classical limit, Mol. Phys. 106, 223 (2008); D. Larese, F. Iachello, A study of quantum phase transitions and quantum monodromy in the bending motion of non-rigid molecules, J. Mol. Struct. 1006, 611 (2011); D. Larese, F. Pérez-Bernal, F. Iachello, Signatures of quantum phase transitions and excited state quantum phase transitions in the vibrational bending dynamics of triatomic molecules, J. Mol. Struct. 1051, 310 (2013).

[5] P. Pérez-Fernández, J.M. Arias, J.E. García-Ramos, and F. Pérez-Bernal, Finite-size corrections in the bosonic algebraic approach to two-dimensional systems, Phys. Rev. A 83, 062125 (2011).

[6] C. Emary and T. Brandes, Quantum Chaos Triggered by Precursors of a Quantum Phase Transition: The Dicke Model, Phys. Rev. Lett. 90, 044101 (2003); Chaos and the quantum phase transition in the Dicke model, Phys. Rev. E 67, 066203 (2003); N. Lambert, C. Emary, and T. Brandes, Entanglement and the Phase Transition in Single-Mode Superradiance, Phys. Rev. Lett. 92, 073602 (2004).

[7] L. Amico, R. Fazio, A. Osterloh, and V. Vedral, Entanglement in many-body systems, Rev. Mod. Phys. 80, 517 (2008).

[8] S. Sachdev, Quantum Phase Transitions (Cambridge University Press, Cambridge, UK, 2011).

[9] C.E. Alonso, J.M. Arias, L. Fortunato, and A. Vitturi, Phase transitions in the interacting boson fermion model: The -unstable case, Phys. Rev. C 72, 061302(R) (2005).

[10] C.E. Alonso, J.M. Arias, and A. Vitturi, Critical-Point Symmetries in Boson-Fermion Systems: The Case of Shape Transitions in Odd Nuclei in a Multiorbit Model, Phys. Rev. Lett. 98, 052501 (2007). 
[11] C.E. Alonso, J.M. Arias, A. Vitturi, Shape phase transition in odd nuclei in a multi-j model: The $U^{B}(6) \otimes U^{F}(12)$ case, Phys. Rev. C 75, 064316 (2007).

[12] J.M. Arias, J.E. García-Ramos, and J. Dukelsky, Phase diagram of the proton-neutron interacting boson model, Phys. Rev. Lett. 93, 212501 (2004).

[13] M.A. Caprio, F. Iachello, Phase structure of the two-fluid proton-neutron system, Phys. Rev. Lett. 93, 242502 (2004).

[14] M.A. Caprio, F. Iachello, Phase structure of a two-fluid bosonic system, Ann. Phys. (N.Y.) 318, 454 (2005).

[15] F. Pérez-Bernal and L. Fortunato, Phase diagram of coupled benders within a $U(3) \times U(3)$ algebraic approach, Phys. Lett. A 376, 236 (2012).

[16] D. Larese, M.A. Caprio, F. Pérez-Bernal, and F. Iachello, A study of the bending motion in tetratomic molecules by the algebraic operator expansion method, J. Chem. Phys. 140, $014304(2014)$.

[17] R.H. Dicke, Coherence in Spontaneous Radiation Processes, Phys. Rev. 93, 99 (1954).

[18] E.T. Jaynes and F.W. Cummings, Comparison of quantum and semiclassical radiation theories with application to the beam maser, Proc. IEEE 51, 89 (1963).

[19] H.J. Lipkin, N. Meshkov, and A.J. Glick, Validity of many-body approximation methods for a solvable model. (I). Exact solutions and perturbation theory, Nucl. Phys. 62, 188 (1965).

[20] F. Iachello, Lie Algebras and Applications (Springer-Verlag, Berlin Heidelberg, Germany, 2006).

[21] J.E. García-Ramos, P. Pérez-Fernández, J.M. Arias, and E. Freire, Phase diagram of the two-fluid Lipkin model: A "butterfly" catastrophe, Phys. Rev. C 93, 034336 (2016).

[22] P. Cejnar, M. Macek, S. Heinze, J. Jolie, and J. Dobeš, Monodromy and excited-state quantum phase transitions in integrable systems: collective vibrations of nuclei, J. Phys. A 39, L515 (2006)

[23] M.A. Caprio, P. Cejnar, and F. Iachello, Excited state quantum phase transitions in manybody systems, Ann. Phys. (N.Y.) 323, 1106 (2008).

[24] A. Frank and P. Van Isacker, Algebraic methods in molecules and nuclei (J. Willey and Sons, New York, 1994).

[25] R.F. Casten and D.D. Warner, The interacting boson approximation, Rev. Mod. Phys. 60, 389 (1988). 
[26] F. Iachello and A. Arima, The Interacting Boson Model (Cambridge University Press, Cambridge, UK, 1987).

[27] J. Jolie, R.F. Casten, P. von Brentano, and V. Werner, Quantum Phase Transition for -Soft Nuclei, Phys. Rev. Lett. 87, 162501 (2001).

[28] P. Stránský, M. Machek, and P. Cejnar, Excited-state quantum phase transitions in systems with two degrees of freedom: Level density, level dynamics, thermal properties, Ann. Phys. (NY) 345, 73 (2014).

[29] P. Stránský, M. Machek, A. Leviatan and P. Cejnar, Excited-state quantum phase transitions in systems with two degrees of freedom: II. Finite-size effects, Ann. Phys. (NY) 356, 57 (2015).

[30] P. Pérez-Fernández, A. Relaño, J.M. Arias, P. Cejnar, J. Dukelsky, and J.E. García-Ramos, Excited-state phase transition and onset of chaos in quantum optical models, Phys. Rev. E 83, 046208 (2011).

[31] M.A. Bastarrachea-Magnani, S. Lerma-Hernández, and J.G. Hirsch, Comparative quantum and semiclassical analysis of atom-field systems. II. Chaos and regularity, Phys. Rev. A 89, $032102(2014)$.

[32] J. Chávez-Carlos, M.A. Bastarrachea-Magnani, S. Lerma-Hernández, and J.G. Hirsch, Classical chaos in atom-field systems, Phys. Rev. E 94, 022209 (2016).

[33] A. Peres, New conserved quantities and test for regular spectra, Phys. Rev. Lett. 53, 1711 (1984).

[34] L.F. Santos and F. Pérez-Bernal, Structure of eigenstates and quench dynamics at an excitedstate quantum phase transition, Phys. Rev. A 92, 050101(R) (2015); L.F. Santos, M. Távora, and F.P. Pérez-Bernal, Excited-state quantum phase transitions in many-body systems with infinite-range interaction: Localization, dynamics, and bifurcation, Phys. Rev. A 94, 012113 (2016).

[35] A. Relaño, J. Dukelsky, P. Pérez-Fernández, and J.M. Arias, Quantum phase transitions of atom-molecule Bose mixtures in a double-well potential, Phys. Rev. E 90, 042139 (2014). 